\title{
THE CLASSIFICATION OF NICHOLS ALGEBRAS OVER GROUPS WITH FINITE ROOT SYSTEM OF RANK TWO
}

\author{
I. HECKENBERGER AND L. VENDRAMIN
}

\begin{abstract}
We classify all groups $G$ and all pairs $(V, W)$ of absolutely simple Yetter-Drinfeld modules over $G$ such that the support of $V \oplus W$ generates $G$, $c_{W, V} c_{V, W} \neq \mathrm{id}$, and the Nichols algebra of the direct sum of $V$ and $W$ admits a finite root system. As a byproduct, we determine the dimensions of such Nichols algebras, and several new families of finite-dimensional Nichols algebras are obtained. Our main tool is the Weyl groupoid of pairs of absolutely simple YetterDrinfeld modules over groups.
\end{abstract}

\section{Contents}

Introduction

1. The examples

2. The Classification Theorem

11

3. Preliminaries

11

4. Reflections of the first pair $\quad 12$

5. Reflections of the second pair 20

6. Reflections of the third pair 25

7. Computing the reflections $\quad 30$

8. Nichols algebras over $\Gamma_{3} \quad 35$

9. Proof of Theorem 2.1 40

References 41

\section{INTRODUCTION}

In the last years, Nichols algebras turned out to be important in many branches of mathematics such as Hopf algebras and quantum groups [30], [7, [27, [32], 34, 35], Schubert calculus [14], 11], and mathematical physics [28], [33]. Nichols algebras appeared first in a work of Nichols [31], where he studies and classifies certain pointed Hopf algebras. Pointed Hopf algebras have applications in conformal field theory [15].

Let $\mathbb{K}$ be a field and let $G$ be a group. The Lifting Method of Andruskiewitsch and Schneider [6] (see also [7]) provides the best known approach to the classification of finite-dimensional pointed Hopf algebras. At the first place, the method 
asks to determine all finite-dimensional Nichols algebras over $G$ and to provide a presentation by generators and relations. Whereas for abelian groups the situation is understood to a great extent [19], 20], 8], [9, 10, less is known for non-abelian groups.

One idea to approach the problem is to adapt the method applied for abelian groups. The problem here is that the structure of the Nichols algebra of a simple Yetter-Drinfeld module over $G$ is very complicated. Only few finite-dimensional examples are known, [18, [21, and for the important examples of Fomin-Kirillov algebras [14] it is not even known whether they are Nichols algebras or whether they are finite-dimensional. Nevertheless, any direct sum of simple Yetter-Drinfeld modules having a finite-dimensional Nichols algebra gives rise to the structure of a Weyl groupoid [5], and surprisingly, the finiteness of the Weyl groupoid implies strong restrictions on the direct summands. Therefore it is reasonable to attack the classification of semi-simple Yetter-Drinfeld modules with finite-dimensional Nichols algebras before looking at the simple objects. The situation is even more astonishing: The functoriality of the Nichols algebra [7, Cor. 2.3] allows to look at Yetter-Drinfeld submodules of simple objects, which are semi-simple with respect to a smaller group. Then information in the semi-simple setting can be used for simple objects [2], 3, 4].

First ideas to analyze in detail the Nichols algebra of a semi-simple YetterDrinfeld module were developed in [22]. That work is based on the notion of the Weyl groupoid of tuples of simple Yetter-Drinfeld modules over arbitrary Hopf algebras with bijective antipode [5], 23], 24]. Using the classification of finite Weyl groupoids of rank two [12, a breakthrough in the approach was achieved in [26].

Recall that for any group $G$, a $\mathbb{K} G$-module $V$ is absolutely simple if $\mathbb{L} \otimes_{\mathbb{K}} V$ is a simple $\mathbb{L} G$-module for any field extension $\mathbb{L}$ of $\mathbb{K}$. We say that a Yetter-Drinfeld module $V$ over a group algebra $\mathbb{K} G$ is absolutely simple, if $\mathbb{L} \otimes_{\mathbb{K}} V$ is a simple YetterDrinfeld module over $\mathbb{L} G$ for any field extension $\mathbb{L}$ of $\mathbb{K}$. Recall from [22] that the groups $\Gamma_{n}$ are central extensions of dihedral groups, whereas the group $T$, defined in [26], is a central extension of $\mathbf{S L}(2,3)$.

Theorem. [26, Thm.4.5]. Let $G$ be a non-abelian group, and $V$ and $W$ be two absolutely simple Yetter-Drinfeld modules over $G$ such that $G$ is generated by the support of $V \oplus W$. Assume that the Nichols algebra of $V \oplus W$ is finite-dimensional. If $c_{W, V} c_{V, W} \neq \mathrm{id}_{V \otimes W}$, then $G$ is an epimorphic image of $T$ or of $\Gamma_{n}$ for $n \in\{2,3,4\}$.

We remark that if the square of the braiding between $V$ and $W$ is the identity, then $\mathfrak{B}(V \oplus W)$ is isomorphic to $\mathfrak{B}(V) \otimes \mathfrak{B}(W)$ as $\mathbb{N}_{0}$-graded objects in ${ }_{G}^{G} \mathcal{Y} \mathcal{D}$ by [16, Thm. 2.2]. On the other hand, the assumption that $G$ is generated by $\operatorname{supp}(V \oplus W)$ is natural since the braiding of $V \oplus W$, and hence the structure of $\mathfrak{B}(V \oplus W)$ as a 
braided Hopf algebra, depends only on the action and coaction of the subgroup of $G$ generated by $\operatorname{supp}(V \oplus W)$.

Already in [22], Nichols algebras of pairs of simple Yetter-Drinfeld modules over non-abelian epimorphic images of $\Gamma_{2}$ were studied and new Nichols algebras of dimension 1296 over fields of characteristic 3 were found. In [25], the Nichols algebras over non-abelian epimorphic images of $T$ and $\Gamma_{4}$ were studied and new Nichols algebras of dimensions 80621568, 262144 (if char $\mathbb{K} \neq 2$ ) and 1259712, 65536 (if char $\mathbb{K}=2$ ) were found. The situation is more complicated when $G$ is a non-abelian epimorphic image of $\Gamma_{3}$, and it is studied in this work. It is the first case where one meets a finite-dimensional Nichols algebra not of diagonal type which has a non-standard Weyl groupoid. We obtain several new families of Nichols algebras, the ranks and dimensions of which can be read off from Table 1.

TABLE 1. Nichols algebras with finite root system of rank two

\begin{tabular}{|c|c|c|c|c|c|}
\hline rank & group & dimension & char $\mathbb{K}$ & support & reference \\
\hline 4 & $\Gamma_{2}$ & 64 & & $Z_{2}^{2,2}$ & Example 1.2 \\
\hline 4 & $\Gamma_{2}$ & 1296 & 3 & $Z_{2}^{2,2}$ & Example 1.3 \\
\hline 4 & $\Gamma_{3}$ & 10368 & $\neq 2,3$ & $Z_{3}^{3,1}$ & Thm. 8.2 \\
\hline 4 & $\Gamma_{3}$ & 5184 & 2 & $Z_{3}^{3,1}$ & Thm. 8.2 \\
\hline 4 & $\Gamma_{3}$ & 1152 & 3 & $Z_{3}^{3,1}$ & Thm. 8.2 \\
\hline 4 & $\Gamma_{3}$ & 2239488 & 2 & $Z_{3}^{3,1}$ & Thms. 8.6. 8.8 \\
\hline 5 & $\Gamma_{3}$ & 10368 & $\neq 2,3$ & $Z_{3}^{3,2}$ & Thm. 8.1 \\
\hline 5 & $\Gamma_{3}$ & 5184 & 2 & $Z_{3}^{3,2}$ & Thm. 8.1 \\
\hline 5 & $\Gamma_{3}$ & 1152 & 3 & $Z_{3}^{3,2}$ & Thm. 8.1 \\
\hline 5 & $\Gamma_{3}$ & 2304 & & $Z_{3}^{3,2}$ & Thm. 8.3 \\
\hline 5 & $\Gamma_{3}$ & 2304 & & $Z_{3}^{3,1}$ & Thm. 8.4 \\
\hline 5 & $\Gamma_{3}$ & 2239488 & 2 & $Z_{3}^{3,2}$ & Thm. 8.7 \\
\hline 5 & $T$ & 80621568 & $\neq 2$ & $Z_{T}^{4,1}$ & Example [.7 \\
\hline 5 & $T$ & 1259712 & 2 & $Z_{T}^{4,1}$ & Example 1.7 \\
\hline 6 & $\Gamma_{4}$ & 262144 & $\neq 2$ & $Z_{4}^{4,2}$ & Example 1.5 \\
\hline 6 & $\Gamma_{4}$ & 65536 & 2 & $Z_{4}^{4,2}$ & Example 1.5 \\
\hline
\end{tabular}

Having studied Nichols algebras over non-abelian epimorphic images of $\Gamma_{2}, \Gamma_{3}$, $\Gamma_{4}$, and $T$, we are able to classify all pairs $(V, W)$ of absolutely simple Yetter-Drinfeld modules over a non-abelian group $G$ such that the Nichols algebra of $V \oplus W$ is finitedimensional. Moreover, we determine the Hilbert series and the decomposition of 
the Nichols algebra of $V \oplus W$ into the tensor product of Nichols algebras of simple Yetter-Drinfeld modules. The finite-dimensional Nichols algebras appearing in our classification are listed in Table 1. The pairs $(V, W)$ of absolutely simple YetterDrinfeld modules over $G$ appear in Section [1, Our main theorem is the following.

Theorem. Let $G$ be a non-abelian group and $V$ and $W$ be two absolutely simple Yetter-Drinfeld modules over $G$ such that $G$ is generated by the support of $V \oplus W$. Assume that $\left(\mathrm{id}-c_{W, V} c_{V, W}\right)(V \otimes W)$ is non-zero and that the Nichols algebra $\mathfrak{B}(V \oplus$ $W)$ is finite-dimensional. Then $\mathfrak{B}(V \oplus W)$ is one of the Nichols algebras of Table 1 .

See Theorem 2.1 for the more precise statement. Let us explain briefly how the proof of Theorem 2.1 goes. We have to study in detail Nichols algebras over nonabelian epimorphic images of the groups $\Gamma_{2}, \Gamma_{3}, \Gamma_{4}$ and $T$. The analysis concerning the group $\Gamma_{2}$ was done in [22] and the groups $\Gamma_{4}$ and $T$ were studied in [25]. The classification of finite-dimensional Nichols algebras associated with $\Gamma_{3}$ is one of the main results of this paper and requires several steps. We need to deal with three different pairs $(V, W)$ of absolutely simple Yetter-Drinfeld modules over non-abelian epimorphic images of $\Gamma_{3}$. We first determine when $(\operatorname{ad} V)^{m}(W)$ and $(\operatorname{ad} W)^{m}(V)$ are absolutely simple or zero and then we compute the Cartan matrix of $(V, W)$. Then we prove that these pairs are essentially the only pairs which we need to consider, and the reflections of these pairs are computed. With this information we compute the finite root systems of rank two associated with Nichols algebras over non-abelian epimorphic images of $\Gamma_{3}$. This information allows us to determine the structure of such Nichols algebras.

The main result of our paper is expected to lead to powerful applications. We intend to attack the classification of finite-dimensional Nichols algebras of finite direct sums of absolutely simple Yetter-Drinfeld modules over groups. For this project it is very useful that the reflections of the absolutely simple pairs are already calculated. On the other hand, we are confident that our classification will be useful to study Nichols algebras over simple Yetter-Drinfeld modules, as it was done for example in [3, 4].

We do not know the defining relations of the Nichols algebras appearing in our classification. In the spirit of [1, Question 5.9], one then has the following problem: Give a nice presentation by generators and relations of the Nichols algebras appearing in Table 1. To attack this, the ideas of [9, 10] could be useful.

The paper is organized as follows. In Section 1 we list all the finite-dimensional Nichols algebras appearing in our classification. In Section 2 we state the main result of the paper, Theorem 2.1. This theorem classifies Nichols algebras of group type over the sum of two absolutely simple Yetter-Drinfeld modules. Sections 4, 4, 5, 
6, 7 and 8 are devoted to understand the structure and the root systems of finitedimensional Nichols algebras over non-abelian epimorphic images of $\Gamma_{3}$. Finally, in Section 9, we prove our main result, Theorem 2.1.

\section{THE EXAMPLES}

Before stating our main result, we collect all the examples of Nichols algebras with finite root systems obtained over non-abelian epimorphic images of $\Gamma_{2}, \Gamma_{3}, \Gamma_{4}$ and $T$. These are the examples which appear in our classification in Theorem 2.1.

Recall from [22] that the group $\Gamma_{n}$ for $n \geq 2$ is the group given by generators $a, b, \nu$ and relations

$$
b a=\nu a b, \quad \nu a=a \nu^{-1}, \quad \nu b=b \nu, \quad \nu^{n}=1,
$$

and $T$ is the group given by generators $\zeta, \chi_{1}, \chi_{2}$ and relations

$$
\zeta \chi_{1}=\chi_{1} \zeta, \quad \zeta \chi_{2}=\chi_{2} \zeta, \quad \chi_{1} \chi_{2} \chi_{1}=\chi_{2} \chi_{1} \chi_{2}, \quad \chi_{1}^{3}=\chi_{2}^{3}
$$

Remark 1.1. The groups $\Gamma_{2}, \Gamma_{3}, \Gamma_{4}$ and $T$ are isomorphic to the enveloping groups of the quandles $Z_{2}^{2,2}, Z_{3}^{3,1}, Z_{3}^{3,2}, Z_{4}^{4,2}$ and $Z_{T}^{4,1}$, see [26, §2] and [25] for an alternative description of these quandles. An epimorphic image $G$ of any of these enveloping groups $G_{X}$ is non-abelian if and only if the restriction of the epimorphism $G_{X} \rightarrow G$ to the quandle $X$ is injective.

By [22], the group $\Gamma_{3}$ is isomorphic to the group given by generators $\nu, \zeta$ and $\gamma$ and relations

$$
\gamma \nu=\nu^{2} \gamma, \quad \zeta \gamma=\gamma \zeta, \quad \zeta \nu=\nu \zeta, \quad \nu^{3}=1
$$

1.1. Epimorphic images of $\Gamma_{2}$. In [22, §4], Nichols algebras over non-abelian epimorphic images of $\Gamma_{2}$ were studied. Let $G$ be a non-abelian group. Let $g, h, \epsilon \in G$, and assume that there is a group epimorphism

$$
\Gamma_{2} \rightarrow G, \quad a \mapsto g, \quad b \mapsto h, \quad \nu \mapsto \epsilon
$$

Example 1.2. Let $V, W \in G_{G}^{G} \mathcal{Y} \mathcal{D}$. Assume that $V \simeq M(g, \rho)$, where $\rho$ is a character of $G^{g}=\left\langle\epsilon, g, h^{2}\right\rangle$, and $W \simeq M(h, \sigma)$, where $\sigma$ is a character of $G^{h}=\left\langle\epsilon, h, g^{2}\right\rangle$. Let $v \in V_{g}$ with $v \neq 0$. Then $\{v, h v\}$ is a basis of $V$ and the degrees of these basis vectors are $g$ and $\epsilon g$, respectively. Similarly, let $w \in W_{h}$ with $w \neq 0$. Then $\{w, g w\}$ is a basis of $W$ and the degrees of these vectors are $h$ and $\epsilon h$, respectively. The action of $G$ on $V$ and $W$ is given by the following tables:

\begin{tabular}{c|cc}
$V$ & $v$ & $h v$ \\
\hline$\epsilon$ & $\rho(\epsilon) v$ & $\rho(\epsilon) h v$ \\
$h$ & $h v$ & $\rho\left(h^{2}\right) v$ \\
$g$ & $\rho(g) v$ & $\rho(\epsilon) \rho(g) h v$
\end{tabular}

\begin{tabular}{c|cc}
$W$ & $w$ & $g w$ \\
\hline$\epsilon$ & $\sigma(\epsilon) w$ & $\sigma(\epsilon) g w$ \\
$h$ & $\sigma(h) w$ & $\sigma(\epsilon) \sigma(h) g w$ \\
$g$ & $g w$ & $\sigma\left(g^{2}\right) w$
\end{tabular}


Assume that

$$
\rho\left(\epsilon h^{2}\right) \sigma\left(\epsilon g^{2}\right)=1, \quad \rho(g)=\sigma(h)=-1 .
$$

Then, by [22, Thm. 4.6], $\operatorname{dim} \mathfrak{B}(V \oplus W)=64$ and the Hilbert series of the Nichols algebra $\mathfrak{B}(V \oplus W)$ is

$$
\mathcal{H}\left(t_{1}, t_{2}\right)=\left(1+t_{1}\right)^{2}\left(1+t_{1} t_{2}\right)^{2}\left(1+t_{2}\right)^{2} .
$$

A special case of this example appeared first in [29, Example 6.5].

Example 1.3. Let $V, W \in{ }_{G}^{G} \mathcal{Y D}$. Assume that $V \simeq M(g, \rho)$, where $\rho$ is a character of $G^{g}=\left\langle\epsilon, g, h^{2}\right\rangle$, and $W \simeq M(h, \sigma)$, where $\sigma$ is a character of $G^{h}=\left\langle\epsilon, h, g^{2}\right\rangle$. Assume also that char $\mathbb{K}=3$ and that

$$
\rho\left(\epsilon h^{2}\right) \sigma\left(\epsilon g^{2}\right)=1, \quad \rho(g)=1, \quad \sigma(h)=-1 .
$$

Then, by [22, Thm. 4.7], $\operatorname{dim} \mathfrak{B}(V \oplus W)=1296$ and the Hilbert series of the Nichols algebra $\mathfrak{B}(V \oplus W)$ is

$$
\mathcal{H}\left(t_{1}, t_{2}\right)=\left(1+t_{1}+t_{1}^{2}\right)^{2}\left(1+t_{2}\right)^{2}\left(1+t_{1} t_{2}+t_{1}^{2} t_{2}^{2}\right)^{2}\left(1+t_{1}^{2} t_{2}\right)^{2} .
$$

Remark 1.4. The braiding of the Yetter-Drinfeld modules of Examples 1.2 and 1.3 can be obtained from the following table:

\begin{tabular}{c|cccc} 
& $v$ & $h v$ & $w$ & $g w$ \\
\hline$g$ & $\rho(g) v$ & $\rho(\epsilon g) h v$ & $g w$ & $\sigma\left(g^{2}\right) w$ \\
$\epsilon g$ & $\rho(\epsilon g) v$ & $\rho(g) h v$ & $\sigma(\epsilon) g w$ & $\sigma\left(\epsilon g^{2}\right) w$ \\
$h$ & $h v$ & $\rho\left(h^{2}\right) v$ & $\sigma(h) w$ & $\sigma(\epsilon h) g w$ \\
$\epsilon h$ & $\rho(\epsilon) h v$ & $\rho\left(\epsilon h^{2}\right) v$ & $\sigma(\epsilon h) w$ & $\sigma(h) g w$
\end{tabular}

1.2. Epimorphic images of $\Gamma_{4}$. Finite-dimensional Nichols algebras over nonabelian epimorphic images of $\Gamma_{4}$ were computed in [25, §5]. Let $G$ be a non-abelian group and let $g, h, \epsilon \in G$. Assume that there is a group epimorphism

$$
\Gamma_{4} \rightarrow G, \quad a \mapsto g, \quad b \mapsto h, \quad \nu \mapsto \epsilon
$$

such that $\epsilon^{2} \neq 1$.

Example 1.5. Let $V, W \in{ }_{G}^{G} \mathcal{Y D}$. Assume that $V \simeq M(h, \rho)$, where $\rho$ is a character of the centralizer $G^{h}=\left\langle\epsilon, h, g^{2}\right\rangle$ with $\rho(h)=-1$. Let $v \in V_{h}$ with $v \neq 0$. Then the elements $v, g v$ form a basis of $V$, and the degrees of these basis vectors are $h$ and $g h g^{-1}=\epsilon^{-1} h$, respectively. The action of $G$ on $V$ is given by the following table:

\begin{tabular}{c|cc}
$V$ & $v$ & $g v$ \\
\hline$\epsilon$ & $\rho(\epsilon) v$ & $\rho(\epsilon)^{-1} g v$ \\
$h$ & $-v$ & $-\rho(\epsilon)^{-1} g v$ \\
$g$ & $g v$ & $\rho\left(g^{2}\right) v$
\end{tabular}

Assume that $W \simeq M(g, \sigma)$, where $\sigma$ is a character of $G^{g}=\left\langle\epsilon^{2}, \epsilon^{-1} h^{2}, g\right\rangle$ with $\sigma(g)=-1$. Let $w \in W_{g}$ with $w \neq 0$. The elements $w, h w, \epsilon w, \epsilon h w$ form a basis of 
$W$. The degrees of these basis vectors are $g, \epsilon g, \epsilon^{2} g$ and $\epsilon^{3} g$, respectively. The action of $G$ on $W$ is given by the following table:

\begin{tabular}{c|cccc}
$W$ & $w$ & $h w$ & $\epsilon w$ & $\epsilon h w$ \\
\hline$\epsilon$ & $\epsilon w$ & $\epsilon h w$ & $\sigma\left(\epsilon^{2}\right) w$ & $\sigma\left(\epsilon^{2}\right) h w$ \\
$h$ & $h w$ & $\sigma\left(\epsilon^{-1} h^{2}\right) \epsilon w$ & $\epsilon h w$ & $\sigma\left(\epsilon h^{2}\right) w$ \\
$g$ & $-w$ & $-\sigma\left(\epsilon^{2}\right) \epsilon h w$ & $-\sigma\left(\epsilon^{2}\right) \epsilon w$ & $-\sigma\left(\epsilon^{2}\right) h w$
\end{tabular}

Assume further that

$$
\rho(\epsilon)=\rho\left(g^{2}\right) \sigma\left(\epsilon^{-1} h^{2}\right), \quad \rho(\epsilon)^{2}=-1 .
$$

Then, by [25, Thm. 5.4],

$$
\mathcal{H}\left(t_{1}, t_{2}\right)=\left(1+t_{2}\right)^{4}\left(1+t_{2}^{2}\right)^{2}\left(1+t_{1} t_{2}\right)^{4}\left(1+t_{1}^{2} t_{2}^{2}\right)^{2} q\left(t_{1} t_{2}^{2}\right) q\left(t_{1}\right),
$$

where

$$
q(t)= \begin{cases}(1+t)^{2}\left(1+t^{2}\right) & \text { if char } \mathbb{K} \neq 2 \\ (1+t)^{2} & \text { if char } \mathbb{K}=2\end{cases}
$$

In particular,

$$
\operatorname{dim} \mathfrak{B}(V \oplus W)= \begin{cases}8^{2} 64^{2}=262144 & \text { if } \operatorname{char} \mathbb{K} \neq 2, \\ 4^{2} 64^{2}=65536 & \text { if } \operatorname{char} \mathbb{K}=2 .\end{cases}
$$

Remark 1.6. The braiding of the Yetter-Drinfeld module of Example 1.5] can be obtained from the following table:

\begin{tabular}{c|cccccc} 
& $v$ & $g v$ & $w$ & $h w$ & $\epsilon w$ & $\epsilon h w$ \\
\hline$h$ & $-v$ & $-\rho(\epsilon)^{-1} g v$ & $h w$ & $\sigma\left(\epsilon^{-1} h^{2}\right) \epsilon w$ & $\epsilon h w$ & $\sigma\left(\epsilon h^{2}\right) w$ \\
$\epsilon^{3} h$ & $-\rho(\epsilon)^{3} v$ & $-g v$ & $\sigma\left(\epsilon^{2}\right) \epsilon h w$ & $\sigma\left(\epsilon^{-1} h^{2}\right) w$ & $h w$ & $\sigma\left(\epsilon^{-1} h^{2}\right) \epsilon w$ \\
$g$ & $g v$ & $\rho\left(g^{2}\right) v$ & $-w$ & $-\sigma\left(\epsilon^{2}\right) \epsilon h w$ & $-\sigma\left(\epsilon^{2}\right) \epsilon w$ & $-\sigma\left(\epsilon^{2}\right) h w$ \\
$\epsilon g$ & $\rho\left(\epsilon^{3}\right) g v$ & $\rho\left(\epsilon g^{2}\right) v$ & $-\epsilon w$ & $-h w$ & $-w$ & $-\sigma\left(\epsilon^{2}\right) \epsilon h w$ \\
$\epsilon^{2} g$ & $\rho\left(\epsilon^{2}\right) g v$ & $\rho\left(\epsilon^{2} g^{2}\right) v$ & $-\sigma\left(\epsilon^{2}\right) w$ & $-\epsilon h w$ & $-\epsilon w$ & $-h w$ \\
$\epsilon^{3} g$ & $\rho(\epsilon) g v$ & $\rho\left(\epsilon^{3} g^{2}\right) v$ & $-\sigma\left(\epsilon^{2}\right) \epsilon w$ & $-\sigma\left(\epsilon^{2}\right) h w$ & $-\sigma\left(\epsilon^{2}\right) w$ & $-\epsilon h w$
\end{tabular}

1.3. Epimorphic images of $T$. Nichols algebras over non-abelian epimorphic images of the group $T$ were studied in [25, §2]. Let $G$ be a non-abelian group, and let $x_{1}, x_{2}, z \in G$. Assume that there is a group epimorphism

$$
T \mapsto G, \quad \zeta \mapsto z, \quad \chi_{1} \mapsto x_{1}, \quad \chi_{2} \mapsto x_{2}
$$

Clearly, $z$ is a central element of $G$. Moreover, the elements

$$
x_{1}, x_{2}, x_{3}:=x_{2} x_{1} x_{2}^{-1}, x_{4}:=x_{1} x_{2} x_{1}^{-1}
$$

form a conjugacy class of $G$.

Example 1.7. Let $V, W \in{ }_{G}^{G} \mathcal{Y D}$. Assume that $V \simeq M(z, \rho)$, where $\rho$ is a character of the centralizer $G^{z}=G$, and $W=M\left(x_{1}, \sigma\right)$, where $\sigma$ is a character of $G^{x_{1}}=$ 
$\left\langle x_{1}, x_{2} x_{3}, z\right\rangle$ with $\sigma\left(x_{1}\right)=-1$ and $\sigma\left(x_{2} x_{3}\right)=1$. Let $v \in V_{z} \backslash\{0\}$. Then $\{v\}$ is basis of $V$. The action of $G$ on $V$ is given by

$$
z v=\rho(z) v, \quad x_{i} v=\rho\left(x_{1}\right) v \quad \text { for all } i \in\{1,2,3,4\} .
$$

Let $w_{1} \in W_{x_{1}}$ such that $w_{1} \neq 0$. Then the vectors

$$
w_{1}, w_{2}:=-x_{4} w_{1}, w_{3}:=-x_{2} w_{1}, w_{4}:=-x_{3} w_{1}
$$

form a basis of $W$. The degrees of these vectors are $x_{1}, x_{2}, x_{3}$ and $x_{4}$, respectively. The action of $G$ on $W$ is given by the following table:

Assume further that

\begin{tabular}{c|cccc}
$W$ & $w_{1}$ & $w_{2}$ & $w_{3}$ & $w_{4}$ \\
\hline$x_{1}$ & $-w_{1}$ & $-w_{4}$ & $-w_{2}$ & $-w_{3}$ \\
$x_{2}$ & $-w_{3}$ & $-w_{2}$ & $-w_{4}$ & $-w_{1}$ \\
$x_{3}$ & $-w_{4}$ & $-w_{1}$ & $-w_{3}$ & $-w_{2}$ \\
$x_{4}$ & $-w_{2}$ & $-w_{3}$ & $-w_{1}$ & $-w_{4}$ \\
$z$ & $\sigma(z) w_{1}$ & $\sigma(z) w_{2}$ & $\sigma(z) w_{3}$ & $\sigma(z) w_{4}$
\end{tabular}

$$
\left(\rho\left(x_{1}\right) \sigma(z)\right)^{2}-\rho\left(x_{1}\right) \sigma(z)+1=0, \quad \rho\left(x_{1} z\right) \sigma(z)=1 .
$$

Then, by [25, Thm. 2.8], $\mathfrak{B}(V \oplus W)$ is finite-dimensional. If char $\mathbb{K} \neq 2$, then

$$
\mathcal{H}\left(t_{1}, t_{2}\right)=(6)_{t_{1}}(6)_{t_{1} t_{2}^{3}}(6)_{t_{1}^{2} t_{2}^{3}}(2)_{t_{2}}^{2}(3)_{t_{2}}(6)_{t_{2}}(2)_{t_{1} t_{2}}^{2}(3)_{t_{1} t_{2}}(6)_{t_{1} t_{2}}(2)_{t_{1} t_{2}^{2}}^{2}(3)_{t_{1} t_{2}^{2}}(6)_{t_{1} t_{2}^{2}}
$$

and $\operatorname{dim} \mathfrak{B}(V \oplus W)=6^{3} 72^{3}=80621568$, and if $\operatorname{char} \mathbb{K}=2$, then

$$
\mathcal{H}\left(t_{1}, t_{2}\right)=(3)_{t_{1}}(3)_{t_{1} t_{2}^{3}}(3)_{t_{1}^{2} t_{2}^{3}}(2)_{t_{2}}^{2}(3)_{t_{2}}^{2}(2)_{t_{1} t_{2}}^{2}(3)_{t_{1} t_{2}}^{2}(2)_{t_{1} t_{2}^{2}}^{2}(3)_{t_{1} t_{2}^{2}}^{2}
$$

and $\operatorname{dim} \mathfrak{B}(V \oplus W)=3^{3} 36^{3}=1259712$.

Remark 1.8. The structure of the Yetter-Drinfeld module of Example 1.7 can be read off the following table:

\begin{tabular}{c|ccccc} 
& $v$ & $w_{1}$ & $w_{2}$ & $w_{3}$ & $w_{4}$ \\
\hline$z$ & $\rho(z) v$ & $\sigma(z) w_{1}$ & $\sigma(z) w_{2}$ & $\sigma(z) w_{3}$ & $\sigma(z) w_{4}$ \\
$x_{1}$ & $\rho\left(x_{1}\right) v$ & $-w_{1}$ & $-w_{4}$ & $-w_{2}$ & $-w_{3}$ \\
$x_{2}$ & $\rho\left(x_{1}\right) v$ & $-w_{3}$ & $-w_{2}$ & $-w_{4}$ & $-w_{1}$ \\
$x_{3}$ & $\rho\left(x_{1}\right) v$ & $-w_{4}$ & $-w_{1}$ & $-w_{3}$ & $-w_{2}$ \\
$x_{4}$ & $\rho\left(x_{1}\right) v$ & $-w_{2}$ & $-w_{3}$ & $-w_{1}$ & $-w_{4}$
\end{tabular}

1.4. Epimorphic images of $\Gamma_{3}$. The results of this section will be proved in Section 8. Let $G$ be a non-abelian group. Let $g, \epsilon, z \in G$, and assume that there is a group epimorphism

$$
\Gamma_{3} \rightarrow G, \quad \gamma \mapsto g, \quad \nu \mapsto \epsilon, \quad \zeta \mapsto z .
$$

Example 1.9. Let $V \in{ }_{G}^{G} \mathcal{Y D}$. Assume that $V \simeq M(g, \rho)$, where $\rho$ is a character of $G^{g}=\langle g, z\rangle$. Let $v \in V_{g}$ with $v \neq 0$. The elements $v, \epsilon v$ and $\epsilon^{2} v$ form a basis of $V$. The degrees of these vectors are $g, g \epsilon$ and $g \epsilon^{2}$, respectively. 
Similarly, let $W \in{ }_{G}^{G} \mathcal{Y D}$ such that $W \simeq M(\epsilon z, \sigma)$, where $\sigma$ is a character of the centralizer $G^{\epsilon}=\left\langle\epsilon, z, g^{2}\right\rangle$. Let $w \in W_{\epsilon z}$ with $w \neq 0$. Then $w, g w$ is a basis of $W$. The degrees of these basis vectors are $\epsilon z$ and $\epsilon^{2} z$, respectively. The actions of $G$ on $V$ and $W$ are given in the following tables:

\begin{tabular}{|c|c|c|c|c|c|c|}
\hline$V$ & $v$ & $\epsilon v$ & $\epsilon^{2} v$ & $W$ & $w$ & $g w$ \\
\hline$\epsilon$ & $\epsilon v$ & $\epsilon^{2} v$ & $v$ & $\epsilon$ & $\sigma(\epsilon) w$ & $\sigma(\epsilon)^{2} g w$ \\
\hline$z$ & $\rho(z) v$ & $\rho(z) \epsilon v$ & $\rho(z) \epsilon^{2} v$ & $z$ & $\sigma(z) w$ & $\sigma(z) g w$ \\
\hline$g$ & $\rho(g) v$ & $\rho(g) \epsilon^{2} v$ & $\rho(g) \epsilon v$ & $g$ & & $\sigma\left(g^{2}\right) w$ \\
\hline
\end{tabular}

If $\rho(g)=\sigma(\epsilon z)=-1, \rho(z)^{2} \sigma\left(\epsilon g^{2}\right)=1$, and $1+\sigma(\epsilon)+\sigma(\epsilon)^{2}=0$, then

$$
\operatorname{dim} \mathfrak{B}(V \oplus W)= \begin{cases}10368 & \text { if char } \mathbb{K} \notin\{2,3\} \\ 5184 & \text { if char } \mathbb{K}=2, \\ 1152 & \text { if char } \mathbb{K}=3,\end{cases}
$$

and

$$
\mathcal{H}\left(t_{1}, t_{2}\right)=(2)_{t_{2}}\left(h_{p}^{\prime}\right)_{t_{2}}(2)_{t_{1} t_{2}}^{2}(3)_{t_{1} t_{2}}\left(h_{p}\right)_{t_{1}^{2} t_{2}}(2)_{t_{1}}^{2}(3)_{t_{1}}
$$

where $p=$ char $\mathbb{K}, h_{2}=3, h_{3}=2$, and $h_{p}=6$ for all $p \notin\{2,3\}$, and $h_{3}^{\prime}=2, h_{p}^{\prime}=6$ for all $p \neq 3$, by Theorem 8.1 .

If $\rho(g)=\sigma(\epsilon z)=-1, \rho(z)^{2} \sigma\left(\epsilon g^{2}\right)=\sigma(\epsilon)=1$, and char $\mathbb{K} \neq 3$, then

$$
\mathcal{H}\left(t_{1}, t_{2}\right)=(2)_{t_{2}}^{2}(2)_{t_{1} t_{2}}^{2}(3)_{t_{1} t_{2}}(2)_{t_{1}^{2} t_{2}}^{2}(2)_{t_{1}}^{2}(3)_{t_{1}}
$$

and $\operatorname{dim} \mathfrak{B}(V \oplus W)=2304$, by Theorem 8.3 .

If char $\mathbb{K}=2, \rho(g)=1,(3)_{\sigma(\epsilon)}=0, \sigma(z)=\sigma(\epsilon)$, and $\rho(z)^{2} \sigma\left(\epsilon g^{2}\right)=1$, then $\operatorname{dim} \mathfrak{B}(V \oplus W)=2239488$ and

$$
\mathcal{H}\left(t_{1}, t_{2}\right)=(3)_{t_{2}}^{2}(2)_{t_{1} t_{2}^{2}}^{2}(3)_{t_{1} t_{2}^{2}}(2)_{t_{1}^{2} t_{2}^{3}}(3)_{t_{1} t_{2}}(4)_{t_{1} t_{2}}(6)_{t_{1} t_{2}}(6)_{t_{1}^{2} t_{2}^{2}}(2)_{t_{1}^{2} t_{2}}(2)_{t_{1}}^{2}(3)_{t_{1}},
$$

by Theorem 8.7 .

Example 1.10. Let $V \in{ }_{G}^{G} \mathcal{Y} \mathcal{D}$. Assume that $V \simeq M(g, \rho)$, where $\rho$ is a character of $G^{g}=\langle g, z\rangle$. Let $v \in V_{g}$ with $v \neq 0$. The elements $v, \epsilon v$ and $\epsilon^{2} v$ form a basis of $V$. The degrees of these vectors are $g, g \epsilon$ and $g \epsilon^{2}$, respectively.

Let $W \in{ }_{G}^{G} \mathcal{Y D}$ such that $W \simeq M(z, \sigma)$, where $\sigma$ is a character of $G$. Let $w \in W_{z}$ with $w \neq 0$. Then $w$ is a basis of $W$. The action of $G$ on $V$ can be obtained from Example 1.9.

Let $p=\operatorname{char} \mathbb{K}$. If $\rho(g)=-1,(3)_{-\rho(z) \sigma(g)}=0$, and $\rho(z) \sigma(g z)=1$, then

$$
\operatorname{dim} \mathfrak{B}(V \oplus W)= \begin{cases}10368 & \text { if char } \mathbb{K} \notin\{2,3\} \\ 5184 & \text { if char } \mathbb{K}=2, \\ 1152 & \text { if char } \mathbb{K}=3,\end{cases}
$$

and

$$
\mathcal{H}\left(t_{1}, t_{2}\right)=\left(h_{p}\right)_{t_{2}}(2)_{t_{1} t_{2}}^{2}(3)_{t_{1} t_{2}}(2)_{t_{1}^{2} t_{2}}\left(h_{p}^{\prime}\right)_{t_{1}^{2} t_{2}}(2)_{t_{1}}^{2}(3)_{t_{1}}
$$

by Theorem 8.2, where $h_{p}$ and $h_{p}^{\prime}$ are as in Example 1.9. 
If $\operatorname{char} \mathbb{K}=2, \sigma(z)=1,(3)_{\rho(z) \sigma(g)}=0$, and $(\rho(g)-1)(\rho(g z) \sigma(g)-1)=0$, then $\operatorname{dim} \mathfrak{B}(V \oplus W)=2239488$, and

$$
\mathcal{H}\left(t_{1}, t_{2}\right)=(2)_{t_{2}}(3)_{t_{1} t_{2}}(4)_{t_{1} t_{2}}(6)_{t_{1} t_{2}}(6)_{t_{1}^{2} t_{2}^{2}}(2)_{t_{1}^{4} t_{2}^{3}}(2)_{t_{1}^{3} t_{2}^{2}}^{2}(3)_{t_{1}^{3} t_{2}^{2}}(3)_{t_{1}^{2} t_{2}}^{2}(2)_{t_{1}}^{2}(3)_{t_{1}}
$$

or

$$
\mathcal{H}\left(t_{1}, t_{2}\right)=(2)_{t_{2}}(2)_{t_{1} t_{2}}^{2}(3)_{t_{1} t_{2}}(3)_{t_{1}^{2} t_{2}}^{2}(2)_{t_{1}^{3} t_{2}}^{2}(3)_{t_{1}^{3} t_{2}}(2)_{t_{1}^{4} t_{2}}(3)_{t_{1}}(4)_{t_{1}}(6)_{t_{1}}(6)_{t_{1}^{2}},
$$

by Theorems 8.6 and 8.8 .

Example 1.11. Let $V \in{ }_{G}^{G} \mathcal{Y D}$. Assume that $V \simeq M(g, \rho)$, where $\rho$ is a character of $G^{g}=\langle g, z\rangle$. Let $v \in V_{g}$ with $v \neq 0$. The elements $v, \epsilon v$ and $\epsilon^{2} v$ form a basis of $V$. The degrees of these vectors are $g, g \epsilon$ and $g \epsilon^{2}$, respectively. The action of $G$ on $V$ can be obtained from Example 1.9.

Let $W \in{ }_{G}^{G} \mathcal{Y D}$ such that $W \simeq M(z, \sigma)$, where $\sigma$ is an absolutely irreducible representation of $G$ of degree $\geq 2$. Then $\operatorname{char} \mathbb{K} \neq 3, \operatorname{deg} \sigma=2, \sigma\left(1+\epsilon+\epsilon^{2}\right)=0$, and the isomorphism class of $W$ is uniquely determined by the constants $\sigma\left(g^{2}\right)$ and $\sigma(z)$, see Lemma 3.2 ,

Assume that $\rho(g)=\sigma(z)=-1$ and $\rho\left(z^{2}\right) \sigma\left(g^{2}\right)=1$. Then

$$
\mathcal{H}\left(t_{1}, t_{2}\right)=(2)_{t_{2}}^{2}(2)_{t_{1} t_{2}}^{2}(3)_{t_{1} t_{2}}(2)_{t_{1}^{2} t_{2}}^{2}(2)_{t_{1}}^{2}(3)_{t_{1}}
$$

and $\operatorname{dim} \mathfrak{B}(V \oplus W)=2304$ by Theorem 8.4 ,

Remark 1.12. The braidings of the Yetter-Drinfeld modules of Examples 1.9, 1.10, and 1.11, respectively, can be obtained from the following tables:

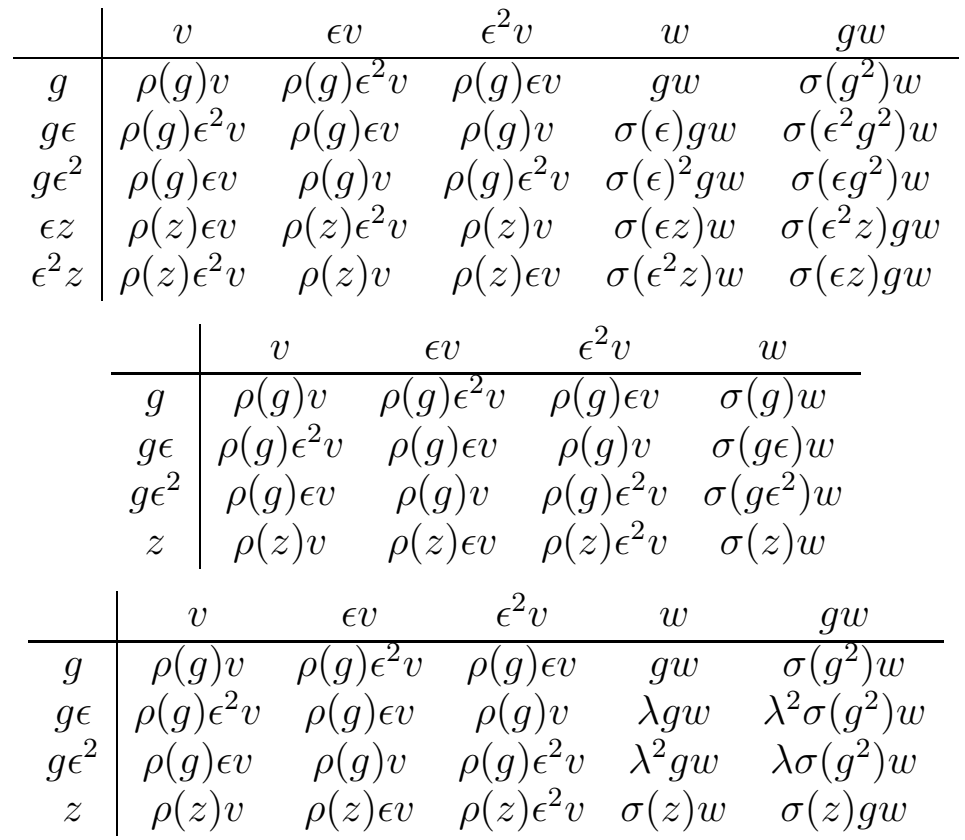




\section{The Classification Theorem}

Now we state the main theorem of the paper. It provides the classification of a class of finite-dimensional Nichols algebras of group type. We list important data of these Nichols algebras in Table 1 .

Theorem 2.1. Let $G$ be a non-abelian group and $V$ and $W$ be two finite-dimensional absolutely simple Yetter-Drinfeld modules over $G$. Assume that $c_{W, V} c_{V, W} \neq \mathrm{id}_{V \otimes W}$ and that the support of $V \oplus W$ generates the group $G$. Then the following are equivalent:

(1) The Nichols algebra $\mathfrak{B}(V \oplus W)$ is finite-dimensional.

(2) The pair $(V, W)$ admits all reflections and the Weyl groupoid of $(V, W)$ is finite.

(3) Up to permutation of its entries, the pair $(V, W)$ is one of the pairs of Examples 1.2, 1.3, 1.5, 1.7, 1.9, 1.10, and 1.11 of Section 1.

In this case, the rank and the dimension of $\mathfrak{B}(V \oplus W)$ appear in Table 1 .

Theorem 2.1 will be proved in Section 9

\section{PRELiminaries}

Let us first state some useful results from [23, 22]. Recall that $S_{n} \in \operatorname{End}\left(V^{\otimes n}\right)$, where $n \in \mathbb{N}$, denotes the quantum symmetrizer.

Lemma 3.1. [22, Thm. 1.1] Let $V$ and $W$ be Yetter-Drinfeld modules over a Hopf algebra $H$ with bijective antipode. Let $\varphi_{0}=0, X_{0}^{V, W}=W$, and

$$
\begin{aligned}
\varphi_{m} & =\mathrm{id}-c_{V \otimes(m-1) \otimes W, V} c_{V, V} \otimes(m-1) \otimes W \\
X_{m}^{V, W} & =\varphi_{m}\left(V \otimes X_{m-1}\right) \subseteq V^{\otimes m} \otimes W
\end{aligned}
$$

for all $m \geq 1$. Then $(\operatorname{ad} V)^{n}(W) \simeq X_{n}^{V, W}$ for all $n \in \mathbb{N}_{0}$.

Now we collect information on $\Gamma_{3}$ which will be needed in the paper. By [22, §3], the center of $\Gamma_{3}$ is $Z\left(\Gamma_{3}\right)=\left\langle\zeta, \gamma^{2}\right\rangle$, and the conjugacy classes of $\Gamma_{3}$ are

$$
\delta^{G}=\{\delta\}, \quad(\nu \delta)^{G}=\left\{\nu \delta, \nu^{2} \delta\right\}, \quad(\gamma \delta)^{G}=\left\{\nu^{j} \gamma \delta \mid 0 \leq j \leq 2\right\},
$$

where $\delta$ is running over all elements of $Z\left(\Gamma_{3}\right)$. In this section, let $G$ be a group. Assume that there exist $g, \epsilon, z \in G, \epsilon \neq 1$, such that there is a group epimorphism $\Gamma_{3} \rightarrow G$ with $\gamma \mapsto g, \nu \mapsto \epsilon$ and $\zeta \mapsto z$. Note that the condition $\epsilon \neq 1$ just means that $G$ is non-abelian.

Lemma 3.2. Assume that $\mathbb{K}$ is algebraically closed. Let $V$ be a simple $\mathbb{K} G$-module and let $\rho: \mathbb{K} G \rightarrow \operatorname{End}(V)$ be the corresponding representation of $\mathbb{K} G$. Then $\operatorname{dim} V \leq$ 2. Moreover, if $\operatorname{dim} V=2$ then $\operatorname{char} \mathbb{K} \neq 3, \rho\left(1+\epsilon+\epsilon^{2}\right)=0$, and the isomorphism class of $V$ is uniquely determined by the scalars $\rho\left(g^{2}\right)$ and $\rho(z)$. 
Proof. Let $v \in V$ be an eigenvector of $\rho(\epsilon)$. Then $V=\mathbb{K} v+\mathbb{K} g v$ and hence $\operatorname{dim} V \leq$ 2.

Assume that $\operatorname{dim} V=2$. Then $g v \notin \mathbb{K} v$. If $\epsilon v=1$, then $\epsilon g v=g \epsilon^{2} v=g v$. Then $\rho(\epsilon)=\operatorname{id}_{V}$, and $\mathbb{K} w$ for an eigenvector $w$ of $\rho(g)$ is a $\mathbb{K} G$-invariant subspace of $V$. This is a contradiction to the simplicity of $V$. Since $\epsilon^{3}=1$ and $1+\epsilon+\epsilon^{2} \in Z(\mathbb{K} G)$, we conclude that $\rho\left(1+\epsilon+\epsilon^{2}\right)=0$ and char $\mathbb{K} \neq 3$. Thus $v, g v$ is a basis of $V$ consisting of eigenvectors of $\rho(\epsilon)$.

Let now $W$ be a simple $\mathbb{K} G$-module with $\operatorname{dim} W=2$, and let $w \in W \backslash\{0\}$ and $\lambda \in \mathbb{K}$ such that $\epsilon v=\lambda v, \epsilon w=\lambda w$. Assume that $g^{2} w=\rho\left(g^{2}\right) w$ and $z w=\rho(z) w$. Then the map $f: V \rightarrow W, v \mapsto w, g v \mapsto g w$, is an isomorphism of $\mathbb{K} G$-modules. This proves the lemma.

\section{Reflections of the First PAir}

Let $G$ be a non-abelian group, and let $g, \epsilon, z \in G$. Assume that there is an epimorphism $\Gamma_{3} \rightarrow G$ with $\gamma \mapsto g, \nu \mapsto \epsilon, \zeta \mapsto z$. Let $V \in{ }_{G}^{G} \mathcal{Y D}$ such that $V \simeq M(g, \rho)$, where $\rho$ is an absolutely irreducible representation of the centralizer $G^{g}=\langle z, g\rangle$. Since this centralizer is abelian, $\operatorname{deg} \rho=1$. Let $v \in V_{g}$ with $v \neq 0$. The elements $v, \epsilon v, \epsilon^{2} v$ form a basis of $V$. The degrees of these basis vectors are $g, g \epsilon$ and $g \epsilon^{2}$, respectively.

Remark 4.1. The action of $G$ on $V$ is given by the following table:

\begin{tabular}{c|ccc}
$M(g, \rho)$ & $v$ & $\epsilon v$ & $\epsilon^{2} v$ \\
\hline$\epsilon$ & $\epsilon v$ & $\epsilon^{2} v$ & $v$ \\
$z$ & $\rho(z) v$ & $\rho(z) \epsilon v$ & $\rho(z) \epsilon^{2} v$ \\
$g$ & $\rho(g) v$ & $\rho(g) \epsilon^{2} v$ & $\rho(g) \epsilon v$
\end{tabular}

Let $W \in{ }_{G}^{G} \mathcal{Y D}$ such that $W \simeq M(\epsilon z, \sigma)$, where $\sigma$ is an absolutely irreducible representation of $G^{\epsilon z}=G^{\epsilon}=\left\langle\epsilon, z, g^{2}\right\rangle$. Since $G^{\epsilon}$ is abelian, $\operatorname{deg} \sigma=1$. Let $w \in W_{\epsilon z}$ with $w \neq 0$. Then $w, g w$ is a basis of $W$. The degrees of these basis vectors are $\epsilon z$ and $\epsilon^{2} z$, respectively.

Remark 4.2. The action of $G$ on $W$ is given by the following table:

\begin{tabular}{c|cc}
$W$ & $w$ & $g w$ \\
\hline$\epsilon$ & $\sigma(\epsilon) w$ & $\sigma(\epsilon)^{2} g w$ \\
$z$ & $\sigma(z) w$ & $\sigma(z) g w$ \\
$g$ & $g w$ & $\sigma\left(g^{2}\right) w$
\end{tabular}

In order to calculate $R_{1}(V, W)$, we first compute the modules $(\operatorname{ad} V)^{n}(W)$ for $n \in \mathbb{N}$. For $n \in \mathbb{N}$ we write $X_{n}=X_{n}^{V, W}$ and $\varphi_{n}=\varphi_{n}^{V, W}$.

Lemma 4.3. The Yetter-Drinfeld module $X_{1}^{V, W}$ is absolutely simple if and only if $\rho(z)^{2} \sigma\left(\epsilon g^{2}\right)=1$. In this case, $X_{1}^{V, W} \simeq M\left(g z, \sigma_{1}\right)$, where $\sigma_{1}$ is the character of 
$G^{g z}=\langle g, z\rangle$ with

$$
\sigma_{1}(g)=-\rho\left(g z^{-1}\right) \sigma(\epsilon), \quad \sigma_{1}(z)=\rho(z) \sigma(z) .
$$

Let $w^{\prime}=\varphi_{1}\left(\epsilon^{2} v \otimes w\right)$. Then $w^{\prime} \in(V \otimes W)_{g z}$ is non-zero. Moreover, $w^{\prime}, \epsilon w^{\prime}$ and $\epsilon^{2} w^{\prime}$ form a basis of $X_{1}^{V, W}$. The degrees of these basis vectors are $g z, g \epsilon z$ and $g \epsilon^{2} z$, respectively.

Proof. By [22, Lemma 1.7], $X_{1}^{V, W} \simeq \mathbb{K} G \varphi_{1}\left(\epsilon^{2} v \otimes w\right)$. Using the actions of $G$ on $V$ and $W$ we obtain that

$$
w^{\prime}=\left(\mathrm{id}-c_{W, V} c_{V, W}\right)\left(\epsilon^{2} v \otimes w\right)=\epsilon^{2} v \otimes w-\rho(z) \sigma(\epsilon)^{2} \epsilon v \otimes g w,
$$

and hence $w^{\prime} \in(V \otimes W)_{g z}$ is non-zero. We compute

$$
\begin{aligned}
g w^{\prime} & =g \epsilon^{2} v \otimes g w-\sigma(\epsilon)^{2} \rho(z) g \epsilon v \otimes g^{2} w \\
& =\rho(g) \epsilon v \otimes g w-\sigma(\epsilon)^{2} \rho(g z) \sigma\left(g^{2}\right) \epsilon^{2} v \otimes w .
\end{aligned}
$$

Since $V$ and $W$ are absolutely simple and $G^{g z}=\langle g\rangle Z(G)$, the Yetter-Drinfeld module $X_{1}^{V, W}$ is absolutely simple if and only if $g w^{\prime} \in \mathbb{K} w^{\prime}$. By the above calculations, this is equivalent to $\rho(z)^{2} \sigma\left(\epsilon g^{2}\right)=1$, and in this case $g w^{\prime}=-\rho\left(g z^{-1}\right) \sigma(\epsilon) w^{\prime}$. The remaining claims are clear.

Now we compute $X_{2}^{V, W}$. Since $(g, g z)$ and $\left(g \epsilon^{2}, g z\right)$ represent the two orbits of $g^{G} \times(g z)^{G}$ under the diagonal action of $G$, we conclude that

$$
X_{2}^{V, W}=\varphi_{2}\left(V \otimes X_{1}^{V, W}\right)=\mathbb{K} G\left\{\varphi_{2}\left(v \otimes w^{\prime}\right), \varphi_{2}\left(\epsilon^{2} v \otimes w^{\prime}\right)\right\} .
$$

For the computation of $\varphi_{2}\left(v \otimes w^{\prime}\right)$ and $\varphi_{2}\left(\epsilon^{2} v \otimes w^{\prime}\right)$ we need the following.

Lemma 4.4. Assume that $\rho(z)^{2} \sigma\left(\epsilon g^{2}\right)=1$. Let $w^{\prime}=\varphi_{1}\left(\epsilon^{2} v \otimes w\right)$. Then

$$
\begin{aligned}
& \varphi_{1}(v \otimes w)=\sigma(\epsilon)^{-1} \epsilon w^{\prime}, \\
& \varphi_{1}(v \otimes g w)=-\rho(z)^{-1} \epsilon^{2} w^{\prime}, \\
& \varphi_{1}\left(\epsilon^{2} v \otimes g w\right)=-\rho(z)^{-1} \sigma(\epsilon)^{-1} \epsilon w^{\prime} .
\end{aligned}
$$

Proof. We first prove Equation (4.3). Since

$$
w^{\prime}=\varphi_{1}\left(\epsilon^{2} v \otimes w\right)=\epsilon^{2} \varphi_{1}(v \otimes \epsilon w)=\epsilon^{2} \sigma(\epsilon) \varphi_{1}(v \otimes w),
$$

Equation (4.3) holds. Now act with $g$ on Equation (4.3) and use Lemma4.3 to obtain Equation (4.4). Finally, to obtain Equation (4.5) act with $\epsilon^{2}$ on Equation (4.4).

Lemma 4.5. Assume that $\rho(z)^{2} \sigma\left(\epsilon g^{2}\right)=1$. Then

$$
\begin{aligned}
& \varphi_{2}\left(\epsilon^{2} v \otimes w^{\prime}\right)=(1+\rho(g))\left(\epsilon^{2} v \otimes w^{\prime}+\rho(g) \sigma(\epsilon) v \otimes \epsilon w^{\prime}\right) \\
& \varphi_{2}\left(v \otimes w^{\prime}\right)=\left(1+\rho(g)^{2} \sigma(\epsilon)\right) v \otimes w^{\prime} \\
&+\rho(g) \sigma(\epsilon)^{2} \epsilon v \otimes \epsilon w^{\prime} \\
&+\rho(g) \sigma(\epsilon)^{2} \epsilon^{2} v \otimes \epsilon^{2} w^{\prime} .
\end{aligned}
$$


In particular, $\varphi_{2}\left(\epsilon^{2} v \otimes w^{\prime}\right)=0$ if and only if $\rho(g)=-1$. Let $w^{\prime \prime}=\varphi_{2}\left(v \otimes w^{\prime}\right)$. Then $w^{\prime \prime} \in(V \otimes V \otimes W)_{g^{2} z}$ is non-zero.

Proof. Recall that $\varphi_{2}=\mathrm{id}-c_{X_{1}^{V, W},{ }^{V}} c_{V, X_{1}^{V, W}}+\left(\mathrm{id} \otimes \varphi_{1}\right) c_{1,2}$. Thus Lemma 4.3 and Equation (4.1) imply that

$$
\begin{aligned}
\varphi_{2}\left(\epsilon^{2} v \otimes w^{\prime}\right)=\epsilon^{2} v \otimes w^{\prime} & +\rho(g)^{2} \sigma(\epsilon) v \otimes \epsilon w^{\prime} \\
& +\rho(g) \epsilon^{2} v \otimes \varphi_{1}\left(\epsilon^{2} v \otimes w\right)-\rho(g z) \sigma(\epsilon)^{2} v \otimes \varphi_{1}\left(\epsilon^{2} v \otimes g w\right) .
\end{aligned}
$$

Hence Equation (4.6) follows from Lemma 4.4. Similarly, we compute

$$
\begin{aligned}
\varphi_{2}\left(v \otimes w^{\prime}\right)= & \left(1+\rho(g)^{2} \sigma(\epsilon)\right) v \otimes w^{\prime} \\
& +\rho(g) \epsilon v \otimes \varphi_{1}(v \otimes w)-\rho(g z) \sigma(\epsilon)^{2} \epsilon^{2} v \otimes \varphi_{1}(v \otimes g w),
\end{aligned}
$$

and then use Lemma 4.4 to obtain Equation (4.7). From this the lemma follows.

Lemma 4.6. Assume that $\rho(z)^{2} \sigma\left(\epsilon g^{2}\right)=1$. Let $w_{1}^{\prime \prime}=\varphi_{2}\left(v \otimes w_{1}^{\prime}\right)$. Then $X_{2}^{V, W}$ is absolutely simple if and only if $\rho(g)=-1$. In this case one of the following holds.

(1) If $\sigma(\epsilon)^{2}+\sigma(\epsilon)+1=0$, then $X_{2}^{V, W}=\mathbb{K} w_{1}^{\prime \prime} \simeq M\left(g^{2} z, \sigma_{2}\right)$, where $\sigma_{2}$ is the character of $G$ given by

$$
\sigma_{2}(g)=-\rho(z)^{-1} \sigma(\epsilon), \quad \sigma_{2}(\epsilon)=1, \quad \sigma_{2}(z)=\rho(z)^{2} \sigma(z) .
$$

(2) If $\sigma(\epsilon)=1$ and char $\mathbb{K} \neq 3$, then $X_{2}^{V, W} \simeq M\left(g^{2} z, \sigma_{2}\right)$, where $\sigma_{2}$ is the twodimensional absolutely irreducible representation of $G$ with basis $\left\{w^{\prime \prime}, \epsilon w^{\prime \prime}\right\}$ and

$$
g w^{\prime \prime}=-\rho(z)^{-1} w^{\prime \prime}, \quad \epsilon^{2} w^{\prime \prime}=-w^{\prime \prime}-\epsilon w^{\prime \prime}, \quad z w^{\prime \prime}=\rho(z)^{2} \sigma(z) w^{\prime \prime} .
$$

Proof. Since $z \in Z(G)$, we conclude that $z w^{\prime \prime}=\rho(z)^{2} \sigma(z) w^{\prime \prime}$. Further,

$$
g w^{\prime \prime}=\varphi_{2}\left(g v \otimes g w^{\prime}\right)=-\rho\left(g^{2} z^{-1}\right) \sigma(\epsilon) w^{\prime \prime}
$$

by Lemma 4.3. Since $g^{2} z$ and $\epsilon g^{2} z$ are not conjugate in $G$, Equation (4.2) and Lemma 4.5 imply that $X_{2}^{V, W}$ is absolutely simple if and only if $\rho(g)=-1$ and $\mathbb{K} G w^{\prime \prime}$ is absolutely simple.

Assume that $\rho(g)=-1$. Since $\epsilon^{3}=1$ in $G$, we know that $\sigma(\epsilon)^{3}=1$. Hence $\sigma(\epsilon)^{2}+\sigma(\epsilon)+1=0$ or $\sigma(\epsilon)=1$. Using Equation (4.7) and Lemma 4.3 one directly computes

$$
(1-\epsilon) w^{\prime \prime}=\left(1+\sigma(\epsilon)+\sigma(\epsilon)^{2}\right)\left(v \otimes w^{\prime}-\epsilon v \otimes \epsilon w^{\prime}\right)
$$

and similarly

$$
\left(1+\epsilon+\epsilon^{2}\right) w^{\prime \prime}=\left(1-\sigma(\epsilon)^{2}\right)^{2}\left(1+\epsilon+\epsilon^{2}\right)\left(v \otimes w^{\prime}\right) .
$$

Suppose first that $\sigma(\epsilon)^{2}+\sigma(\epsilon)+1=0$. Then Equation (4.8) becomes $(1-\epsilon) w^{\prime \prime}=0$ and hence the claim follows. 
Suppose now that $\sigma(\epsilon)=1$ and char $\mathbb{K} \neq 3$. Then $\left\{w^{\prime \prime}, \epsilon w^{\prime \prime}\right\}$ is linearly independent and $\left(1+\epsilon+\epsilon^{2}\right) w^{\prime \prime}=0$ by Equation (4.9). Hence the lemma follows.

Lemma 4.7. Assume that $\rho(z)^{2} \sigma\left(\epsilon g^{2}\right)=1, \rho(g)=-1$. Then $X_{3}^{V, W}=0$.

Proof. Lemma4.6implies that $X_{3}^{V, W}=\mathbb{K} G \varphi_{3}\left(\epsilon v \otimes X_{2}^{V, W}\right)$. Now observe that $\epsilon^{2} g(\epsilon v \otimes$ $\left.w^{\prime \prime}\right) \in \mathbb{K} \epsilon v \otimes \epsilon^{2} w^{\prime \prime}$, and hence it is enough to prove that $\varphi_{3}\left(\epsilon v \otimes w^{\prime \prime}\right)=0$.

Since $\rho(g)=-1$, Equation (4.6) implies that $\varphi_{2}\left(\epsilon^{2} v \otimes w^{\prime}\right)=0$. Act on this equation by $g$ and by $\epsilon^{2}$ and use Lemma 4.3 to conclude that $\varphi_{2}\left(\epsilon v \otimes w^{\prime}\right)=0$ and $\varphi_{2}\left(\epsilon v \otimes \epsilon^{2} w^{\prime}\right)=0$. A direct calculation using Lemmas 4.5 and 4.6 then shows that

$$
\begin{aligned}
\varphi_{3}\left(\epsilon v \otimes w^{\prime \prime}\right)= & \epsilon v \otimes w^{\prime \prime}+\sigma(\epsilon) \epsilon v \otimes \epsilon^{2} w^{\prime \prime}+(1+\sigma(\epsilon)) g \epsilon v \otimes \varphi_{2}\left(\epsilon v \otimes w^{\prime}\right) \\
& \quad-\sigma(\epsilon)^{2} g \epsilon^{2} v \otimes \varphi_{2}\left(\epsilon v \otimes \epsilon w^{\prime}\right)-\sigma(\epsilon)^{2} g v \otimes \varphi_{2}\left(\epsilon v \otimes \epsilon^{2} w^{\prime}\right) \\
= & \epsilon v \otimes\left(w^{\prime \prime}+\sigma(\epsilon) \epsilon^{2} w^{\prime \prime}+\sigma(\epsilon)^{2} \epsilon w^{\prime \prime}\right) .
\end{aligned}
$$

By Lemma 4.6, if $\sigma(\epsilon)^{2}+\sigma(\epsilon)+1=0$ then $\epsilon w^{\prime \prime}=w^{\prime \prime}$, and otherwise $\sigma(\epsilon)=1$ and $\left(1+\epsilon+\epsilon^{2}\right) w^{\prime \prime}=0$. Hence $\varphi_{3}\left(\epsilon v \otimes w^{\prime \prime}\right)=0$.

Now we compute the modules $(\operatorname{ad} W)^{n}(V)$ for $n \in \mathbb{N}$. For $n \in \mathbb{N}$ let $\varphi_{n}=\varphi_{n}^{W, V}$.

Let $X_{1}^{W, V}=\varphi_{1}(W \otimes V)$. By [22, Lemma 1.7], $X_{1}^{W, V}=\mathbb{K} G \varphi_{1}(w \otimes \epsilon v)$. Let $v_{1}^{\prime}=$ $\varphi_{1}(w \otimes \epsilon v)$. A direct calculation yields

$$
v_{1}^{\prime}=w \otimes \epsilon v-\rho(z) \sigma\left(\epsilon^{2}\right) g w \otimes \epsilon^{2} v .
$$

Hence $v_{1}^{\prime} \in(W \otimes V)_{g z}$ is non-zero.

Lemma 4.8. Assume that $\rho(z)^{2} \sigma\left(\epsilon g^{2}\right)=1$. Then $X_{1}^{W, V}$ is an absolutely simple Yetter-Drinfeld module, and $X_{1}^{W, V} \simeq M\left(g z, \rho_{1}\right)$, where $\rho_{1}=\sigma_{1}$. Moreover, $v_{1}^{\prime}, \epsilon v_{1}^{\prime}$, $\epsilon^{2} v_{1}^{\prime}$ is a basis of $X_{1}^{W, V}$, and the degrees of these basis vectors are $g z, g \epsilon z$, and $g \epsilon^{2} z$, respectively.

Proof. Since $X_{1}^{V, W} \simeq X_{1}^{W, V}$ via $c_{V, W}$, the claim follows from Lemma 4.3.

Lemma 4.9. Assume that $\rho(z)^{2} \sigma\left(\epsilon g^{2}\right)=1$ and $\rho(g)=-1$. Then $X_{2}^{W, V}=0$ if and only if $\sigma(\epsilon z)=-1$. Moreover, $X_{2}^{W, V}$ is absolutely simple if and only if $\sigma(\epsilon z) \neq-1$ and $\sigma\left(\epsilon z^{2}\right)=1$. In this case $X_{2}^{W, V} \simeq M\left(g z^{2}, \rho_{2}\right)$, where $\rho_{2}$ is the character of $G^{g}$ given by

$$
\rho_{2}(g)=-\rho(z)^{-2} \sigma(z)^{-1}, \quad \rho_{2}(z)=\rho(z) \sigma(z)^{2} .
$$

Proof. First we act with $\epsilon$ and $\epsilon^{2}$ on $v_{1}^{\prime}=\varphi_{1}(w \otimes \epsilon v)$ to obtain the following formulas:

$$
\varphi_{1}\left(w \otimes \epsilon^{2} v\right)=\sigma(\epsilon)^{2} \epsilon v_{1}^{\prime}, \quad \varphi_{1}(w \otimes v)=\sigma(\epsilon) \epsilon^{2} v_{1}^{\prime} .
$$


Since $G$ acts transitively on $(\epsilon z)^{G} \times(g z)^{G}$ via the diagonal action, we obtain from [22, Lemma 1.7] that $X_{2}^{W, V}=\mathbb{K} G \varphi_{2}\left(w \otimes \epsilon v_{1}^{\prime}\right)$. We compute

$$
\begin{aligned}
\varphi_{2}\left(w \otimes \epsilon v_{1}^{\prime}\right)=w \otimes \epsilon v_{1}^{\prime}-\rho(z) \sigma\left(\epsilon^{2} z^{2}\right) g w \otimes \epsilon^{2} v_{1}^{\prime} \\
\quad+\sigma\left(\epsilon^{2} z\right) w \otimes \varphi_{1}\left(w \otimes \epsilon^{2} v\right)-\rho(z) \sigma(z) g w \otimes \varphi_{1}(w \otimes v) .
\end{aligned}
$$

Equations (4.11) imply that

$$
\varphi_{2}\left(w \otimes \epsilon v_{1}^{\prime}\right)=(1+\sigma(\epsilon z))\left(w \otimes \epsilon v_{1}^{\prime}-\rho(z) \sigma(\epsilon z) g w \otimes \epsilon^{2} v_{1}^{\prime}\right) .
$$

Hence $\varphi_{2}\left(w \otimes \epsilon v_{1}^{\prime}\right)=0$ if and only if $\sigma(\epsilon z)=-1$. Let

$$
v_{1}^{\prime \prime}=w \otimes \epsilon v_{1}^{\prime}-\rho(z) \sigma(\epsilon z) g w \otimes \epsilon^{2} v_{1}^{\prime} .
$$

Then $v_{1}^{\prime \prime} \in\left(W \otimes X_{1}^{W, V}\right)_{g z^{2}}$ is non-zero. Since $\rho(z)^{2} \sigma\left(\epsilon g^{2}\right)=1$,

$$
\begin{aligned}
g v_{1}^{\prime \prime} & =g w \otimes \epsilon^{2} g v_{1}^{\prime}-\rho(z) \sigma(\epsilon z) \sigma(g)^{2} w \otimes \epsilon g v_{1}^{\prime} \\
& =g w \otimes \rho_{1}(g) \epsilon^{2} v_{1}^{\prime}-\rho(z)^{-1} \sigma(z) \rho_{1}(g) w \otimes \epsilon v_{1}^{\prime} \\
& =-\rho_{1}(g) \rho(z)^{-1} \sigma(z)\left(w \otimes \epsilon v_{1}^{\prime}-\rho(z) \sigma(z)^{-1} g w \otimes \epsilon^{2} v_{1}^{\prime}\right) .
\end{aligned}
$$

Thus $X_{2}^{W, V}$ is absolutely simple if and only if $\sigma(\epsilon z) \neq 1$ and $g v_{1}^{\prime \prime} \in \mathbb{K} v_{1}^{\prime \prime}$. This is equivalent to $\sigma(\epsilon z) \neq 1$ and $\sigma\left(\epsilon z^{2}\right)=1$. Finally, the equation $z v_{1}^{\prime}=\rho(z) \sigma(z)^{2} v_{1}^{\prime}$ follows from $v_{1}^{\prime} \in W \otimes W \otimes V$ and $z \in Z(G)$.

Lemma 4.10. Assume that $\rho(z)^{2} \sigma\left(\epsilon g^{2}\right)=1, \rho(g)=-1, \sigma(\epsilon z) \neq-1$ and $\sigma\left(\epsilon z^{2}\right)=$ 1. Define inductively $y_{0}=v$ and

$$
y_{n}=w \otimes \epsilon y_{n-1}-\rho(z) \sigma\left(z^{3 n-1}\right) g w \otimes \epsilon^{2} y_{n-1}
$$

for all $n \geq 1$. Then $y_{n} \in\left(W^{\otimes n} \otimes V\right)_{g z^{n}}$ and

$$
\varphi_{n}\left(w \otimes \epsilon y_{n-1}\right)=\left(1+\sigma(z)^{-1} \cdots+\sigma(z)^{-n+1}\right) y_{n}
$$

for all $n \geq 1$.

Proof. We proceed by induction on $n$. For $n=1$ the claim holds by Equation (4.10) and since $\sigma(\epsilon)^{2}=\sigma(\epsilon)^{-1}=\sigma\left(z^{2}\right)$. It is also clear that $y_{n} \in\left(W^{\otimes n} \otimes V\right)_{g z^{n}}$ for all $n \geq 0$.

Assume that Equation (4.13) holds for some $n \geq 1$. Apply $\epsilon$ and $\epsilon^{2}$ to Equation (4.13) to obtain

$$
\begin{aligned}
\varphi_{n}\left(w \otimes \epsilon^{2} y_{n-1}\right) & =\left(1+\sigma(z)^{-1}+\cdots+\sigma(z)^{-n+1}\right) \sigma(\epsilon)^{2} \epsilon y_{n} \\
\varphi_{n}\left(w \otimes y_{n-1}\right) & =\left(1+\sigma(z)^{-1}+\cdots+\sigma(z)^{-n+1}\right) \sigma(\epsilon) \epsilon^{2} y_{n} .
\end{aligned}
$$

Since $\sigma\left(\epsilon z^{2}\right)=1$,

$$
\begin{aligned}
& \varphi_{n+1}(w \otimes\left.\epsilon y_{n}\right)=w \otimes \epsilon y_{n}-\rho(z) \sigma(z)^{2 n} \sigma(\epsilon)^{2} g w \otimes \epsilon^{2} y_{n} \\
&+\sigma\left(\epsilon^{2} z\right) w \otimes \varphi_{n}\left(w \otimes \epsilon^{2} y_{n-1}\right)-\rho(z) \sigma\left(z^{3 n-1}\right) \sigma(\epsilon z) g w \otimes \varphi_{n}\left(w \otimes y_{n-1}\right) .
\end{aligned}
$$


Using Equations (4.14) and (4.15) and $\sigma\left(\epsilon z^{2}\right)=1$ one obtains

$$
\varphi_{n+1}\left(w \otimes \epsilon y_{n}\right)=\left(1+\sigma(z)^{-1}+\cdots+\sigma(z)^{-n}\right) y_{n+1},
$$

as desired.

Lemma 4.11. Assume that $\rho(z)^{2} \sigma\left(\epsilon g^{2}\right)=1, \rho(g)=-1, \sigma(\epsilon z) \neq-1$, and $\sigma\left(\epsilon z^{2}\right)=$ 1. Then $X_{n}^{W, V} \simeq M\left(g z^{n}, \rho_{n}\right)$ for all $n \geq 1$, where $\rho_{n}$ is the character of $G^{g}$ given by

$$
\rho_{n}(g)=(-1)^{n+1} \rho(z)^{-n} \sigma(z)^{(3 n+5) n / 2}, \quad \rho_{n}(z)=\rho(z) \sigma(z)^{n} .
$$

Moreover, $X_{n}^{W, V}=0$ if and only if $(n)_{\sigma(z)}^{!}=0$.

Proof. For all $n \geq 0$ let $y_{n}$ be as in Lemma 4.10. Then $z y_{n}=\rho(z) \sigma(z)^{n} y_{n}$, since $y_{n} \in W^{\otimes n} \otimes V$ and $z \in Z(G)$. To prove that $g y_{n}=\rho_{n}(g) y_{n}$ for all $n \geq 1$ we proceed by induction. For $n=1$ this equation holds by Lemmas 4.8 and 4.3 . Suppose now that $g y_{n}=\rho_{n}(g) y_{n}$ for some $n \geq 1$. Then

$$
\begin{aligned}
g y_{n+1} & =g w \otimes \epsilon^{2} g y_{n}-\rho(z) \sigma\left(z^{3 n+2}\right) \sigma\left(g^{2}\right) w \otimes \epsilon g y_{n} \\
& =\rho_{n}(g)\left(g w \otimes \epsilon^{2} y_{n}-\rho(z) \sigma\left(z^{3 n+2}\right) \sigma\left(g^{2}\right) w \otimes \epsilon y_{n}\right) .
\end{aligned}
$$

Since $\sigma\left(\epsilon z^{2}\right)=1$ and $\rho(z)^{2} \sigma\left(\epsilon g^{2}\right)=1$, we conlude that the expression for $\rho_{n+1}(g)$ given in the claim can be written as

$$
\rho_{n+1}(g)=-\rho_{n}(g) \rho(z)^{-1} \sigma\left(z^{3 n-2}\right) .
$$

This implies the claim.

To complete the proof of the lemma we observe that

$$
X_{n}^{W, V}=\mathbb{K} G \varphi_{n}\left(w \otimes \epsilon y_{n}\right)
$$

and hence Equation (4.13) of Lemma 4.10 implies that $X_{n}^{W, V}=0$ if and only if $(k)_{\sigma(z)}=0$ for some $k \leq n$, which is equivalent to say that $(n)_{\sigma(z)}^{!}=0$.

We collect all the results of this section in the following proposition. We write $a_{i, j}^{(V, W)}$ for the entries of the Cartan matrix of the pair $(V, W)$.

Proposition 4.12. Let $V, W \in{ }_{G}^{G} \mathcal{Y D}$ such that $V \simeq M(g, \rho)$, where $\rho$ is a character of $G^{g}$, and $W \simeq M(\epsilon z, \sigma)$, where $\sigma$ is a character of $G^{\epsilon z}$. Then the following hold:

(1) $(\operatorname{ad} V)^{m}(W)$ and $(\operatorname{ad} W)^{m}(V)$ are absolutely simple or zero for all $m \in \mathbb{N}_{0}$ if and only if

$$
\rho(z)^{2} \sigma\left(\epsilon g^{2}\right)=1, \quad \rho(g)=-1, \quad(\sigma(\epsilon z)+1)\left(\sigma\left(\epsilon z^{2}\right)-1\right)=0 .
$$

In particular, these equations imply that $\sigma(z)^{6}=1$.

(2) Assume that the equations for $\rho$ and $\sigma$ in (1) hold. Then the Cartan matrix of $(V, W)$ satisfies $a_{1,2}^{(V, W)}=-2$ and $X_{2}^{V, W} \simeq M\left(g^{2} z, \sigma_{2}\right)$, where

(a) if $1+\sigma(\epsilon)+\sigma(\epsilon)^{2}=0$, then $\sigma_{2}$ is the character of $G$ given by $\sigma_{2}(\epsilon)=1$, $\sigma_{2}(g)=-\rho(z)^{-1} \sigma(\epsilon), \sigma_{2}(z)=\rho(z)^{2} \sigma(z)$, and 
(b) if $\sigma(\epsilon)=1$ and char $\mathbb{K} \neq 3$, then $\sigma_{2}$ is a two-dimensional absolutely irreducible representation of $G$ with $\sigma_{2}\left(g^{2}\right)=\rho(z)^{-2}$ and $\sigma_{2}(z)=\rho(z)^{2} \sigma(z)$.

Moreover,

$$
a_{2,1}^{(V, W)}= \begin{cases}-1 & \text { if } \sigma(\epsilon z)=-1 \\ -2 & \text { if } \sigma(z)=\sigma(\epsilon) \text { and }(3)_{\sigma(\epsilon)}=0 \\ -5 & \text { if } \sigma(z)+\sigma(\epsilon)=(3)_{\sigma(\epsilon)}=0 \text { and char } \mathbb{K} \neq 2,3 \\ 1-p & \text { if char } \mathbb{K}=p \geq 5 \text { and } \sigma(\epsilon)=\sigma(z)=1\end{cases}
$$

and otherwise $(\operatorname{ad} W)^{m}(V) \neq 0$ for all $m \in \mathbb{N}_{0}$. In these cases $X_{m}^{W, V} \simeq$ $M\left(g z^{m}, \rho_{m}\right)$ for $m=-a_{2,1}^{(V, W)}$, where $\rho_{m}$ is the character of $G^{g}$ with $\rho_{1}(z)=$ $\rho(z) \sigma(z), \rho_{1}(g)=\rho(z)^{-1} \sigma(\epsilon)$ and

$$
\rho_{m}(z)=\rho(z) \sigma(z)^{m}, \quad \rho_{m}(g)=(-1)^{m+1} \rho(z)^{-m} \sigma(z)^{(3 m+5) m / 2}
$$

for all $m \geq 2$.

Proof. The first claim follows from Lemmas 4.3, 4.6, 4.7, 4.8, 4.9 and 4.11, Further, Lemmas 4.6 and 4.7 yield the claim concerning $a_{1,2}^{(V, W)}$. Now we prove the claim concerning $a_{2,1}^{(V, W)}$. Since $(\sigma(\epsilon z)+1)\left(\sigma\left(\epsilon z^{2}\right)-1\right)=0$ and $\sigma(\epsilon)^{3}=1$, we need to consider the following cases:

(1) $\sigma(\epsilon z)=-1$ and $\sigma(\epsilon)^{3}=1$,

(2) $\sigma(\epsilon)=\sigma(z)$ and $\sigma(\epsilon)^{2}+\sigma(\epsilon)+1=0$,

(3) $\sigma(\epsilon)=\sigma(z)=1$ and char $\mathbb{K}=p \geq 5$,

(4) $\sigma(\epsilon)=\sigma(z)=1$ and char $\mathbb{K}=0$,

(5) $\sigma(\epsilon)=-\sigma(z), \sigma(\epsilon)^{2}+\sigma(\epsilon)+1=0$ and $\operatorname{char} \mathbb{K} \neq 2,3$.

Using the equivalence between $(\operatorname{ad} W)^{m}(V)=0$ and $(m)_{\sigma(z)}^{!}=0$ of Lemma 4.11, one easily obtains the second claim.

Corollary 4.13. Let $V, W \in{ }_{G}^{G} \mathcal{Y D}$ with $V \simeq M(g, \rho)$ and $W \simeq M(\epsilon z, \sigma)$, where $\rho$ is a character of $G^{g}$ and $\sigma$ is a character of $G^{\epsilon}$. Assume that

$$
\rho(g)=\sigma(\epsilon z)=-1, \quad \rho(z)^{2} \sigma\left(\epsilon g^{2}\right)=1, \quad(3)_{\sigma(\epsilon)}=0 .
$$

Let $g^{\prime}=g^{-1}, \epsilon^{\prime}=\epsilon, z^{\prime}=g^{2} z, \rho^{\prime}$ be the representation of $G^{g^{\prime}}$ dual to $\rho$, and $\sigma^{\prime}$ be the character of $G$ given by $\sigma^{\prime}(g)=-\rho(z)^{-1} \sigma(\epsilon), \sigma^{\prime}(\epsilon)=1, \sigma^{\prime}(z)=\rho(z)^{2} \sigma(z)$. Then $a_{1,2}^{(V, W)}=-2$ and

$$
R_{1}(V, W)=\left(V^{*}, X_{2}^{V, W}\right)
$$

with $V^{*} \simeq M\left(g^{\prime}, \rho^{\prime}\right), X_{2}^{V, W} \simeq M\left(z^{\prime}, \sigma^{\prime}\right)$ and

$$
\rho^{\prime}\left(g^{\prime}\right)=-1, \quad \rho^{\prime}\left(z^{\prime}\right) \sigma^{\prime}\left(z^{\prime} g^{\prime}\right)=1, \quad 1-\rho^{\prime}\left(z^{\prime}\right) \sigma^{\prime}\left(g^{\prime}\right)+\rho^{\prime}\left(z^{\prime}\right)^{2} \sigma^{\prime}\left(g^{\prime}\right)^{2}=0 .
$$


Proof. Using Proposition 4.12 one obtains that $a_{1,2}^{(V, W)}=-2$ and hence the description of $R_{1}(V, W)$ follows. It is clear that $\rho^{\prime}\left(g^{\prime}\right)=\rho(g)=-1$. A direct calculation yields

$$
\begin{aligned}
& \rho^{\prime}\left(z^{\prime}\right) \sigma^{\prime}\left(z^{\prime} g^{\prime}\right)=-\sigma(\epsilon z)=1, \\
& 1-\rho^{\prime}\left(z^{\prime}\right) \sigma^{\prime}\left(g^{\prime}\right)+\rho^{\prime}\left(z^{\prime}\right)^{2} \sigma^{\prime}\left(g^{\prime}\right)^{2}=1+\sigma(\epsilon)^{-1}+\sigma(\epsilon)^{-2}=0 .
\end{aligned}
$$

This completes the proof.

Corollary 4.14. Let $V, W \in{ }_{G}^{G} \mathcal{Y D}$ with $V \simeq M(g, \rho)$ and $W \simeq M(\epsilon z, \sigma)$, where $\rho$ is a character of $G^{g}$ and $\sigma$ is a character of $G^{\epsilon}$. Assume that

$$
\rho(g)=\sigma(\epsilon z)=-1, \quad \rho(z)^{2} \sigma\left(\epsilon g^{2}\right)=1, \quad(3)_{\sigma(\epsilon)}=0 .
$$

Let $g^{\prime \prime}=g z, \epsilon^{\prime \prime}=\epsilon^{-1}, z^{\prime \prime}=z^{-1}, \rho^{\prime \prime}$ be the character of $G^{g}$ given by $\rho^{\prime \prime}(g)=$ $\rho(z)^{-1} \sigma(\epsilon)$ and $\rho^{\prime \prime}(z)=\rho(z) \sigma(z)$, and $\sigma^{\prime \prime}$ be the representation of $G^{\epsilon z}$ dual to $\sigma$. Then $a_{2,1}^{(V, W)}=-1$ and

$$
R_{2}(V, W)=\left(X_{1}^{W, V}, W^{*}\right)
$$

with $X_{1}^{W, V} \simeq M\left(g^{\prime \prime}, \rho^{\prime \prime}\right), W^{*} \simeq M\left(\epsilon^{\prime \prime} z^{\prime \prime}, \sigma^{\prime \prime}\right)$, and

$$
\rho^{\prime \prime}\left(g^{\prime \prime}\right)=\sigma^{\prime \prime}\left(\epsilon^{\prime \prime} z^{\prime \prime}\right)=-1, \quad \rho^{\prime \prime}\left(z^{\prime \prime}\right)^{2} \sigma^{\prime \prime}\left(\epsilon^{\prime \prime} g^{\prime \prime 2}\right)=1, \quad(3)_{\sigma^{\prime \prime}\left(\epsilon^{\prime \prime}\right)}=0 .
$$

Proof. The assumptions on $\rho$ and $\sigma$ and Proposition 4.12 yield $a_{2,1}^{(V, W)}=-1$ and hence the description of $R_{2}(V, W)$ follows. Then we compute

$$
\begin{aligned}
& \sigma^{\prime \prime}\left(\epsilon^{\prime \prime} z^{\prime \prime}\right)=\sigma^{\prime \prime}\left(\epsilon^{-1} z^{-1}\right)=\sigma(\epsilon z)=-1, \\
& \rho^{\prime \prime}\left(g^{\prime \prime}\right)=\rho^{\prime \prime}(g z)=\rho^{\prime \prime}(g) \rho^{\prime \prime}(z)=-1, \\
& 1+\sigma^{\prime \prime}\left(\epsilon^{\prime \prime}\right)+\sigma^{\prime \prime}\left(\epsilon^{\prime \prime}\right)^{2}=1+\sigma(\epsilon)+\sigma(\epsilon)^{2}=0 .
\end{aligned}
$$

Using that $g$ and $z$ commute, we obtain that

$$
\rho^{\prime \prime}\left(z^{\prime \prime}\right)^{2} \sigma^{\prime \prime}\left(\epsilon^{\prime \prime} g^{\prime \prime 2}\right)=\rho^{\prime \prime}(z)^{-2} \sigma^{\prime \prime}\left(\epsilon^{-1} g^{2} z^{2}\right)=\rho(z)^{-2} \sigma(\epsilon) \sigma(g)^{-2} \sigma(z)^{-4} .
$$

Since $\sigma(\epsilon z)=-1$ we obtain that $\sigma\left(\epsilon z^{-4}\right)=\sigma(\epsilon)^{-1}$. Hence we conclude that

$$
\rho^{\prime \prime}\left(z^{\prime \prime}\right)^{2} \sigma^{\prime \prime}\left(\epsilon^{\prime \prime} g^{\prime \prime 2}\right)=\left(\rho(z)^{2} \sigma\left(\epsilon g^{2}\right)\right)^{-1}=1 .
$$

This completes the proof.

Corollary 4.15. Let $V, W \in{ }_{G}^{G} \mathcal{Y D}$ with $V \simeq M(g, \rho)$ and $W \simeq M(\epsilon z, \sigma)$, where $\rho$ is a character of $G^{g}$ and $\sigma$ is a character of $G^{\epsilon}$. Assume that char $\mathbb{K} \neq 3$ and

$$
\rho(g)=-1, \quad \rho\left(z^{2}\right) \sigma\left(\epsilon g^{2}\right)=1, \quad \sigma(\epsilon)=1, \quad \sigma(z)=-1 .
$$

Further, let $g^{\prime}=g^{-1}, \epsilon^{\prime}=\epsilon, z^{\prime}=g^{2} z$, let $\rho^{\prime}$ be the irreducible representation of $G^{g}$ dual to $\rho$, and let $\sigma^{\prime}$ be an absolutely irreducible representation of $G$ with $\operatorname{deg} \sigma^{\prime}=2$, $\sigma^{\prime}\left(g^{2}\right)=\rho(z)^{-2}$, and $\sigma^{\prime}(z)=-\rho(z)^{2}$. Then $a_{1,2}^{(V, W)}=-2$ and

$$
R_{1}(V, W)=\left(V^{*}, X_{2}^{V, W}\right)
$$


with $V^{*} \simeq M\left(g^{\prime}, \rho^{\prime}\right), X_{2}^{V, W} \simeq M\left(z^{\prime}, \sigma^{\prime}\right)$, and

$$
\rho^{\prime}\left(g^{\prime}\right)=-1, \quad \rho^{\prime}\left(z^{\prime}\right)^{2} \sigma^{\prime}\left(g^{\prime 2}\right)=1, \quad \sigma^{\prime}\left(z^{\prime}\right)=-1 .
$$

Proof. It is similar to the proof of Corollary 4.13.

Corollary 4.16. Let $V, W \in{ }_{G}^{G} \mathcal{Y D}$ with $V \simeq M(g, \rho)$ and $W \simeq M(\epsilon z, \sigma)$, where $\rho$ is a character of $G^{g}$ and $\sigma$ is a character of $G^{\epsilon}$. Assume that char $\mathbb{K} \neq 3$ and

$$
\rho(g)=-1, \quad \rho(z)^{2} \sigma\left(\epsilon g^{2}\right)=1, \quad \sigma(\epsilon)=1, \quad \sigma(z)=-1 .
$$

Further, let $g^{\prime \prime}=g z, \epsilon^{\prime \prime}=\epsilon^{-1}, z^{\prime \prime}=z^{-1}$, let $\rho^{\prime \prime}$ be the character of $G^{g}$ given by $\rho^{\prime \prime}(z)=-\rho(z)$ and $\rho^{\prime \prime}(g)=\rho(z)^{-1}$, and let $\sigma^{\prime \prime}$ be the character of $G^{\epsilon}$ dual to $\sigma$. Then $a_{2,1}^{(V, W)}=-1$ and

$$
R_{2}(V, W)=\left(X_{1}^{W, V}, W^{*}\right)
$$

with $X_{1}^{W, V} \simeq M\left(g^{\prime \prime}, \rho^{\prime \prime}\right), W^{*} \simeq M\left(\epsilon^{\prime \prime} z^{\prime \prime}, \sigma^{\prime \prime}\right)$, and

$$
\rho^{\prime \prime}\left(g^{\prime \prime}\right)=-1, \quad \rho^{\prime \prime}\left(z^{\prime \prime}\right)^{2} \sigma^{\prime \prime}\left(\epsilon^{\prime \prime} g^{\prime \prime 2}\right)=1, \quad \sigma^{\prime \prime}\left(\epsilon^{\prime \prime}\right)=1, \quad \sigma^{\prime \prime}\left(z^{\prime \prime}\right)=-1 .
$$

Proof. It is similar to the proof of Corollary 4.14.

\section{REFLECTIONS OF THE SECOND PAIR}

In this section we have to deal with an irreducible representation of $G$ of degree two. Therefore we assume that $\mathbb{K}$ is algebraically closed. This will not be relevant for our classification of Nichols algebras.

Let $G$ be a non-abelian group, and let $g, \epsilon, z \in G$. Assume that there is an epimorphism $\Gamma_{3} \rightarrow G$ with $\gamma \mapsto g, \nu \mapsto \epsilon, \zeta \mapsto z$. Let $V, W \in{ }_{G}^{G} \mathcal{Y D}$ such that $V \simeq M(g, \rho)$ and $W \simeq M(z, \sigma)$, where $\rho$ is a character of $G^{g}$ and $\sigma$ is a twodimensional irreducible representation of $G$. Let $\lambda$ be an eigenvalue of $\sigma(\epsilon)$ and let $w$ be a corresponding eigenvector. Then, by Lemma 3.2 , char $\mathbb{K} \neq 3$ and $1+\lambda+\lambda^{2}=0$. Hence $\epsilon g w=\lambda^{-1} g w, \lambda^{-1} \neq \lambda$, and $\{w, g w\}$ is a basis of $W_{z}=W$.

Remark 5.1. By the above discussion, we obtain the following table for the action of $G$ on $W$ :

\begin{tabular}{c|cc}
$W$ & $w$ & $g w$ \\
\hline$\epsilon$ & $\lambda w$ & $\lambda^{2} g w$ \\
$z$ & $\sigma(z) w$ & $\sigma(z) g w$ \\
$g$ & $g w$ & $\sigma\left(g^{2}\right) w$
\end{tabular}

Now we compute the modules $(\operatorname{ad} V)^{m}(W)$ for $m \in \mathbb{N}$. First we obtain that $X_{1}^{V, W}=\varphi_{1}(V \otimes W)=\mathbb{K} G \varphi_{1}(v \otimes w)$ and

$$
\varphi_{1}(v \otimes w)=v \otimes(w-\rho(z) g w) .
$$

Hence $w^{\prime}:=\varphi_{1}(v \otimes w) \in(V \otimes W)_{g z}$ is non-zero. 
Lemma 5.2. The Yetter-Drinfeld module $X_{1}^{V, W}$ is simple if and only if $\rho(z)^{2} \sigma\left(g^{2}\right)=$ 1. In this case, $X_{1}^{V, W} \simeq M\left(g z, \sigma_{1}\right)$, where $\sigma_{1}$ is the character of $G^{g}$ with

$$
\sigma_{1}(z)=\rho(z) \sigma(z), \quad \sigma_{1}(g)=-\rho\left(g z^{-1}\right) .
$$

A basis for $X_{1}^{V, W}$ is given by $\left\{w^{\prime}, \epsilon w^{\prime}, \epsilon^{2} w^{\prime}\right\}$. The degrees of these basis vectors are $g z, g \in z$ and $g \epsilon^{2} z$, respectively.

Proof. Since $X_{1}^{V, W}=\mathbb{K} G w^{\prime}$ and $w^{\prime} \in(V \otimes W)_{g z}, X_{1}^{V, W}$ is simple if and only if $g w^{\prime}=\mathbb{K} w^{\prime}$. Since

$$
g w^{\prime}=g v \otimes\left(g w-\rho(z) g^{2} w\right)=\rho(g) v \otimes\left(-\rho(z) \sigma\left(g^{2}\right) w+g w\right),
$$

the latter is equivalent to $g w^{\prime}=-\rho(g z) \sigma\left(g^{2}\right) w^{\prime}, \rho(z)^{2} \sigma\left(g^{2}\right)=1$. From this the claim follows.

Remark 5.3. The action of $G$ on $X_{1}^{V, W}$ can be displayed in a table similar to the one in Remark 4.1, where $v$ has to be replaced by $w^{\prime}$ and $\rho$ has to be replaced by $\sigma_{1}$.

Lemma 5.4. Assume that $\rho(z)^{2} \sigma\left(g^{2}\right)=1$. Then $X_{2}^{V, W} \neq 0$, and $X_{2}^{V, W}$ is simple if and only $\rho(g)=-1$. In this case, $X_{2}^{V, W} \simeq M\left(\epsilon g^{2} z, \sigma_{2}\right)$, where $\sigma_{2}$ is the character of $G^{\epsilon}$ given by

$$
\sigma_{2}(\epsilon)=1, \quad \sigma_{2}(z)=\rho(z)^{2} \sigma(z), \quad \sigma_{2}\left(g^{2}\right)=\rho(z)^{-2} .
$$

The set $\left\{w^{\prime \prime}, g w^{\prime \prime}\right\}$ is a basis of $X_{2}^{V, W}$. The degrees of these vectors are $\epsilon g^{2} z$ and $\epsilon^{2} g^{2} z$, respectively.

Proof. Observe that $X_{2}^{V, W}$ is the direct sum $\mathbb{K} G \varphi_{2}\left(v \otimes w^{\prime}\right) \oplus \mathbb{K} G \varphi_{2}\left(\epsilon^{2} v \otimes w^{\prime}\right)$ of two Yetter-Drinfeld submodules. Acting with $g$ on Equation (5.1) and using Lemma 5.2 we obtain that

$$
\varphi_{1}(v \otimes g w)=-\rho(z)^{-1} w^{\prime} .
$$

A direct computation shows that

$$
\begin{aligned}
\varphi_{2}\left(v \otimes w^{\prime}\right)=v \otimes w^{\prime} & +\rho\left(g^{2}\right) v \otimes w^{\prime} \\
& +\rho(g) v \otimes \varphi_{1}(v \otimes w)-\rho(g z) v \otimes \varphi_{1}(v \otimes g w) .
\end{aligned}
$$

Then $\varphi_{2}\left(v \otimes w^{\prime}\right)=(1+\rho(g))^{2} v \otimes w^{\prime}$. To compute $\varphi_{2}\left(\epsilon^{2} v \otimes w^{\prime}\right)$ we act with $\epsilon^{2}$ on Equations (5.1) and (5.2) to obtain

$$
\varphi_{1}\left(\epsilon^{2} v \otimes w\right)=\lambda \epsilon^{2} w^{\prime}, \quad \varphi_{1}\left(\epsilon^{2} v \otimes g w\right)=-\lambda^{2} \rho(z)^{-1} \epsilon^{2} w^{\prime} .
$$

Then one computes

$$
\begin{aligned}
\varphi_{2}\left(\epsilon^{2} v \otimes w^{\prime}\right)=\epsilon^{2} v \otimes w^{\prime}+\rho(g)^{2} v \otimes \epsilon w^{\prime} & +\rho(g) \epsilon v \otimes \varphi_{1}\left(\epsilon^{2} v \otimes w\right) \\
& -\rho(z) \rho(g) \epsilon v \otimes \varphi_{1}\left(\epsilon^{2} v \otimes g w\right),
\end{aligned}
$$


and hence

$$
\varphi_{2}\left(\epsilon^{2} v \otimes w^{\prime}\right)=\epsilon^{2} v \otimes w^{\prime}+\rho\left(g^{2}\right) v \otimes \epsilon w^{\prime}-\rho(g) \epsilon v \otimes \epsilon^{2} w^{\prime} .
$$

Therefore $w^{\prime \prime}:=\varphi_{2}\left(\epsilon^{2} v \otimes w^{\prime}\right) \in(V \otimes V \otimes W)_{\epsilon g^{2} z}$ is non-zero. We conclude that $\mathbb{K} G \varphi_{2}\left(v \otimes w^{\prime}\right)=0$, that is, $\rho(g)=-1$. In this case one easily obtains from Equation (5.3) that $\epsilon w^{\prime \prime}=w^{\prime \prime}$. The equations $g^{2} w^{\prime \prime}=\rho(z)^{-2} w^{\prime \prime}$ and $z w^{\prime \prime}=\rho(z)^{2} \sigma(z) w^{\prime \prime}$ are clear since $g^{2}, z \in Z(G)$ and $\sigma\left(g^{2}\right)=\rho(z)^{-2}$.

Lemma 5.5. Assume that $\rho(z)^{2} \sigma\left(g^{2}\right)=1$ and $\rho(g)=-1$. Then $X_{3}^{V, W}=0$.

Proof. Act with $\epsilon$ and with $g \epsilon$ on $w^{\prime \prime}=\varphi_{2}\left(\epsilon^{2} v \otimes w^{\prime}\right)$ and use Lemmas 5.4 and 5.2 to obtain that $w^{\prime \prime}=\varphi_{2}\left(v \otimes \epsilon w^{\prime}\right)$ and $g w^{\prime \prime}=-\rho(z)^{-1} \varphi_{2}\left(v \otimes \epsilon^{2} w^{\prime}\right)$, respectively. Now we calculate

$$
\begin{aligned}
\varphi_{3}\left(v \otimes w^{\prime \prime}\right)=v \otimes w^{\prime \prime} & -\rho(z) \epsilon^{2} v \otimes g w^{\prime \prime}-\epsilon v \otimes \varphi_{2}\left(v \otimes w^{\prime}\right) \\
& -v \otimes \varphi_{2}\left(v \otimes \epsilon w^{\prime}\right)-\epsilon^{2} v \otimes \varphi_{2}\left(v \otimes \epsilon^{2} w^{\prime}\right),
\end{aligned}
$$

and hence $\varphi_{3}\left(v \otimes w^{\prime \prime}\right)=0$. Since $X_{3}^{V, W}=\mathbb{K} G \varphi_{3}\left(v \otimes w^{\prime \prime}\right)$, the proof of the lemma is completed.

Now we compute the modules $(\operatorname{ad} W)^{n}(V)$ for $n \in \mathbb{N}$. For $n \in \mathbb{N}$ we write $\varphi_{n}=\varphi_{n}^{W, V}$.

First note that

$$
X_{1}^{W, V}=\varphi_{1}(W \otimes V)=\mathbb{K} G \varphi_{1}(w \otimes v)
$$

Further,

$$
v_{1}^{\prime}:=\varphi_{1}(w \otimes v)=(w-\rho(z) g w) \otimes v \in(W \otimes V)_{g z}
$$

is non-zero.

Lemma 5.6. Assume that $\rho(z)^{2} \sigma\left(g^{2}\right)=1$. Then $X_{1}^{W, V}$ is simple and $X_{1}^{W, V} \simeq$ $M\left(g z, \rho_{1}\right)$, where $\rho_{1}$ is the character of $G^{g z}$ defined by

$$
\rho_{1}(g)=-\rho\left(g z^{-1}\right), \quad \rho_{1}(z)=\rho(z) \sigma(z) .
$$

The set $\left\{v_{1}^{\prime}, \epsilon v_{1}^{\prime}, \epsilon^{2} v_{1}^{\prime}\right\}$ is a basis of $X_{1}^{W, V}$. The degrees of these basis vectors are $g z$, $g \epsilon z$, and $g \epsilon^{2} z$, respectively.

Proof. This follows from Lemma 5.2.

Lemma 5.7. Assume that $\rho(z)^{2} \sigma\left(g^{2}\right)=1$ and $\rho(g)=-1$. Then the following hold:

(1) $X_{2}^{W, V}=0$ if and only if $\sigma(z)=-1$.

(2) $X_{2}^{W, V}$ is simple if and only if $\sigma(z)=1$ and $\sigma(z) \neq-1$. In this case, $X_{2}^{W, V} \simeq$ $M\left(g z^{2}, \rho_{2}\right)$, where $\rho_{2}$ is the character of $G^{g}$ with

$$
\rho_{2}(g)=-\rho(z)^{-2}, \quad \rho_{2}(z)=\rho(z) .
$$


Proof. Since $X_{2}^{W, V}=\varphi_{2}\left(W \otimes X_{1}^{W, V}\right)=\mathbb{K} G \varphi_{2}\left(w \otimes v_{1}^{\prime}\right)$, we need to compute $\varphi_{2}\left(w \otimes v_{1}^{\prime}\right)$. Using Equation (5.4) we obtain that

$$
\begin{aligned}
\varphi_{2}\left(w \otimes v_{1}^{\prime}\right) & =w \otimes v_{1}^{\prime}-c_{X_{1}^{W, V}, W} c_{W, X_{1}^{W, V}}\left(w \otimes v_{1}^{\prime}\right)+\left(\mathrm{id} \otimes \varphi_{1}\right) c_{1,2}\left(w \otimes v_{1}^{\prime}\right) \\
& =(1+\sigma(z))\left(w \otimes v_{1}^{\prime}-\rho(z) \sigma(z) g w \otimes v_{1}^{\prime}\right),
\end{aligned}
$$

and hence the first claim follows. Now assume that $\sigma(z) \neq-1$ and let $v_{1}^{\prime \prime}:=(w-$ $\rho(z) \sigma(z) g w) \otimes v_{1}^{\prime}$. Then $v_{1}^{\prime \prime} \in\left(X_{2}^{W, V}\right)_{g z^{2}}$ is non-zero. Further, using Lemma 5.6] we obtain that

$$
g v_{1}^{\prime \prime}=\left(g w-\rho(z) \sigma(z) g^{2} w\right) \otimes g v_{1}^{\prime}=\rho(z)^{-1}\left(g w-\rho(z) \sigma\left(g^{2} z\right) w\right) \otimes v_{1}^{\prime} .
$$

Observe that $g v_{1}^{\prime \prime}=\mathbb{K} v_{1}^{\prime \prime}$ if and only if $\sigma\left(z^{2}\right)=1$, and then $g v_{1}^{\prime \prime}=-\sigma\left(g^{2} z\right) v_{1}^{\prime \prime}$. Thus $X_{2}^{W, V}$ is simple if and only if $\sigma(z) \neq-1$ and $\sigma(z)=1$. In this case, since $\sigma(z)=1$, we conclude that $X_{2}^{W, V} \simeq M\left(g z^{2}, \rho_{2}\right)$, where $\rho_{2}(z)=\rho(z)$ and $\rho_{2}(g)=-\rho(z)^{-2}$.

Now we define $y_{n}=(w-\rho(z) g w)^{\otimes n} \otimes v$ for all $n \geq 0$.

Lemma 5.8. Assume that $\rho(z)^{2} \sigma\left(g^{2}\right)=1, \rho(g)=-1, \sigma(z)=1$, and $\sigma(z) \neq-1$. Let $n \geq 1$. Then $\varphi_{n}\left(w \otimes y_{n-1}\right)=n y_{n}$ and $X_{n}^{W, V}=n ! \mathbb{K} G y_{n}$. Moreover, if $n ! \neq 0$ then $X_{n}^{W, V} \simeq M\left(g z^{n}, \rho_{n}\right)$, where $\rho_{n}$ is the character on $G^{g}$ given by

$$
\rho_{n}(g)=(-1)^{n+1} \rho(z)^{-n}, \quad \rho_{n}(z)=\rho(z) .
$$

Proof. It is clear that $y_{n} \in\left(W^{\otimes n} \otimes V\right)_{g z^{n}}$ for all $n \in \mathbb{N}$. Moreover,

$$
g(w-\rho(z) g w)=g w-\rho(z)^{-1} w=-\rho(z)^{-1}(w-\rho(z) g w),
$$

and hence $g y_{n}=(-1)^{n+1} \rho(z)^{-n} y_{n}$ for all $n \in \mathbb{N}$. To prove the other claims we proceed by induction on $n$. For $n=1$ the claim holds by Lemma 5.6. So assume that the claim is valid for some $n \geq 1$. Then

$$
X_{n+1}^{W, V}=\varphi_{n+1}\left(w \otimes X_{n}^{W, V}\right)=\varphi_{n+1}\left(w \otimes n ! y_{n}\right) .
$$

Since $\sigma(z)=1$, we obtain that

$$
\varphi_{n+1}\left(w \otimes y_{n}\right)=w \otimes y_{n}-\rho(z) g w \otimes y_{n}+(w-\rho(z) g w) \otimes \varphi_{n}\left(w \otimes y_{n-1}\right) .
$$

Since $\varphi_{n}\left(w \otimes y_{n-1}\right)=n y_{n}$ by induction hypothesis, the latter equation implies that $\varphi_{n+1}\left(w \otimes y_{n}\right)=(n+1) y_{n+1}$. Therefore $X_{n+1}^{W, V}=(n+1) ! \mathbb{K} G y_{n+1}$. The remaining claims are clear.

In the remaining part of this section we do not need and do not use any assumption on the field $\mathbb{K}$.

Proposition 5.9. Let $V, W \in{ }_{G}^{G} \mathcal{Y D}$ such that $V \simeq M(g, \rho)$, where $\rho$ is a character of $G^{g}$, and $W \simeq M(z, \sigma)$, where $\sigma$ is an absolutely irreducible representation of $G$ of degree two. Then char $\mathbb{K} \neq 3$ and the following hold: 
(1) $(\operatorname{ad} V)^{m}(W)$ and $(\operatorname{ad} W)^{m}(V)$ are absolutely simple or zero for all $m \in \mathbb{N}_{0}$ if and only if

$$
\rho(z)^{2} \sigma\left(g^{2}\right)=1, \quad \rho(g)=-1, \quad \sigma(z)^{2}=1 .
$$

(2) Assume that the conditions on $\rho, \sigma$ in (1) hold. Then the Cartan matrix of $(V, W)$ satisfies $a_{1,2}^{(V, W)}=-2$ and $X_{2}^{V, W} \simeq M\left(\epsilon g^{2} z, \sigma_{2}\right)$, where $\sigma_{2}$ is the character of $G^{\epsilon}$ given by

$$
\sigma_{2}(\epsilon)=1, \quad \sigma_{2}(z)=\rho(z)^{2} \sigma(z), \quad \sigma_{2}\left(g^{2}\right)=\sigma\left(g^{2}\right) .
$$

Moreover,

$$
a_{2,1}^{(V, W)}= \begin{cases}-1 & \text { if } \sigma(z)=-1, \\ 1-p & \text { if } \sigma(z)=1 \text { and } \operatorname{char} \mathbb{K}=p \geq 5,\end{cases}
$$

and $X_{m}^{W, V} \simeq M\left(g z^{n}, \rho_{m}\right)$, where $m=-a_{2,1}^{(V, W)}$ and $\rho_{m}$ is the character of $G^{g}$ given by

$$
\rho_{m}(g)=(-1)^{m+1} \rho(z)^{-m}, \quad \rho_{m}(z)=\rho(z) \sigma(z) .
$$

Proof. Since $W$ is absolutely simple and $z \in Z(G)$, the representation $\sigma$ is absolutely irreducible. Hence char $\mathbb{K} \neq 3$ and $\sigma\left(1+\epsilon+\epsilon^{2}\right)=0$ by Lemma 3.2.

Since $\left(\operatorname{ad}\left(\mathbb{L} \otimes_{\mathbb{K}} V\right)\right)^{m}\left(\mathbb{L} \otimes_{\mathbb{K}} W\right) \simeq \mathbb{L} \otimes_{\mathbb{K}}(\operatorname{ad} V)^{m}(W)$ for all $m \in \mathbb{N}$ and all field extensions $\mathbb{L}$ of $\mathbb{K}$, for (1) we may assume that $\mathbb{K}$ is algebraically closed. Thus (1) follows from Lemmas $5.2,5.4,5.5,5.6,5.7$, and 5.8. Further, under the conditions on $\rho$ and $\sigma$ in (1) we obtain that $a_{12}^{(V, W)}=-2$ by Lemmas 5.4 and 5.5, and the structure of $X_{2}^{V, W}$ is given by Lemma 5.4. Similarly, the value of $a_{21}^{(V, W)}$ and the structure of $X_{m}^{W, V}$ are obtained from Lemmas 5.7 and 5.8 .

Corollary 5.10. Assume that char $\mathbb{K} \neq 3$. Let $V, W \in{ }_{G}^{G} \mathcal{Y D}$ such that $V \simeq M(g, \rho)$ and $W \simeq M(z, \sigma)$, where $\rho$ is a character of $G^{g}$, and $\sigma$ is an absolutely irreducible representation of $G$ of degree two. Assume that

$$
\rho(z)^{2} \sigma\left(g^{2}\right)=1, \quad \rho(g)=-1, \quad \sigma(z)=-1 .
$$

Further, let $g^{\prime}=g^{-1}, \epsilon^{\prime}=\epsilon, z^{\prime}=g^{2} z, \rho^{\prime}$ be the character of $G^{g^{\prime}}=G^{g}$ dual to $\rho$, and $\sigma^{\prime}$ be the character of $G^{\epsilon}$ given by $\sigma^{\prime}(\epsilon)=1, \sigma^{\prime}(z)=-\rho(z)^{2}, \sigma^{\prime}\left(g^{2}\right)=\sigma\left(g^{2}\right)$. Then $a_{1,2}^{(V, W)}=-2$ and

$$
R_{1}(V, W)=\left(V^{*}, X_{2}^{V, W}\right)
$$

with $V^{*} \simeq M\left(g^{\prime}, \rho^{\prime}\right), X_{2}^{V, W} \simeq M\left(\epsilon^{\prime} z^{\prime}, \sigma^{\prime}\right)$, and

$$
\rho^{\prime}\left(g^{\prime}\right)=-1, \quad \sigma^{\prime}\left(z^{\prime}\right)=-1, \quad \rho^{\prime}\left(z^{\prime}\right)^{2} \sigma^{\prime}\left(\epsilon^{\prime} g^{\prime 2}\right)=1, \quad \sigma^{\prime}\left(\epsilon^{\prime}\right)=1 .
$$


Proof. By Proposition 5.9 we obtain that $a_{1,2}^{(V, W)}=-2$ and hence the description of $R_{1}(V, W)$ follows. Then

$$
\sigma^{\prime}\left(z^{\prime}\right)=\sigma^{\prime}\left(g^{2}\right) \sigma^{\prime}(z)=-\sigma\left(g^{2}\right) \rho\left(z^{2}\right)=-1 .
$$

Similarly one proves the other formulas.

Corollary 5.11. Assume that char $\mathbb{K} \neq 3$. Let $V, W \in{ }_{G}^{G} \mathcal{Y D}$ such that $V \simeq M(g, \rho)$ and $W \simeq M(z, \sigma)$, where $\rho$ is a character of $G^{g}$, and $\sigma$ is an absolutely irreducible representation of $G$ of degree two. Assume that

$$
\rho(z)^{2} \sigma\left(g^{2}\right)=1, \quad \rho(g)=-1, \quad \sigma(z)=-1 .
$$

Let $g^{\prime \prime}=g z, \epsilon^{\prime \prime}=\epsilon^{-1}, z^{\prime \prime}=z^{-1}, \rho^{\prime \prime}$ be the character of $G^{g}$ given by $\rho^{\prime \prime}(g)=\rho(z)^{-1}$ and $\rho^{\prime \prime}(z)=-\rho(z)$, and $\sigma^{\prime \prime}$ be the degree two representation of $G$ dual to $\sigma$. Then $a_{2,1}^{(V, W)}=-1$ and

$$
R_{2}(V, W)=\left(X_{1}^{W, V}, W^{*}\right)
$$

with $X_{1}^{W, V} \simeq M\left(g^{\prime \prime}, \rho^{\prime \prime}\right), W^{*} \simeq M\left(z^{\prime \prime}, \sigma^{\prime \prime}\right)$, and

$$
\rho^{\prime \prime}\left(z^{\prime \prime}\right)^{2} \sigma^{\prime \prime}\left(g^{\prime \prime 2}\right)=1, \quad \rho^{\prime \prime}\left(g^{\prime \prime}\right)=-1, \quad \sigma^{\prime \prime}\left(z^{\prime \prime}\right)=-1 .
$$

Proof. It is similar to the proof of Corollary 5.10 .

\section{REFLECTIONS OF THE THIRD PAIR}

Let $V, W \in{ }_{G}^{G} \mathcal{Y D}$ such that $V \simeq M(g, \rho)$, where $\rho$ is a character of $G^{g}$, and $W \simeq M(z, \sigma)$, where $\sigma$ is a character of $G$. Let $w \in W=W_{z}$ with $w \neq 0$. Then $\{w\}$ is a basis of $W$. Since $g \epsilon=\epsilon^{-1} g$ and $\epsilon^{3}=1$, we obtain that

$$
g w=\sigma(g) w, \quad \epsilon w=w, \quad z w=\sigma(z) w .
$$

We first compute the modules $(\operatorname{ad} V)^{m}(W)$ for $m \in \mathbb{N}$.

Lemma 6.1. The Yetter-Drinfeld module $X_{1}^{V, W}$ is non-zero if and only if $\rho(z) \sigma(g) \neq$ 1. In this case, $X_{1}^{V, W}$ is absolutely simple and $X_{1}^{V, W} \simeq M\left(g z, \sigma_{1}\right)$, where $\sigma_{1}$ is the character of $G^{g}$ given by

$$
\sigma_{1}(g)=\rho(g) \sigma(g), \quad \sigma_{1}(z)=\rho(z) \sigma(z) .
$$

Let $w^{\prime}=v \otimes w$. Then $\left\{w^{\prime}, \epsilon w^{\prime}, \epsilon^{2} w^{\prime}\right\}$ is a basis of $X_{1}^{V, W}$. The degrees of these basis vectors are $g z, g \epsilon z$, and $g \epsilon^{2} z$, respectively.

Again, the action of $G$ on $X_{1}^{V, W}$ can be displayed in a table similar to the one in Remark 4.1, where $v$ has to be replaced by $w^{\prime}$ and $\rho$ has to be replaced by $\sigma_{1}$. 
Proof. Write $X_{1}^{V, W}=\varphi_{1}(V \otimes W)=\mathbb{K} G \varphi_{1}(v \otimes w)$ and compute

$$
\varphi_{1}(v \otimes w)=v \otimes w-c_{W, V} c_{V, W}(v \otimes w)=(1-\rho(z) \sigma(g)) v \otimes w .
$$

Then $w^{\prime}=v \otimes w \in(V \otimes W)_{g z}$ is non-zero and $X_{1}^{V, W}=0$ if and only if $\rho(z) \sigma(g)=1$. Assume that $\rho(z) \sigma(g) \neq 1$. Then

$$
g w^{\prime}=\rho(g) \sigma(g) w^{\prime}, \quad z w^{\prime}=\rho(z) \sigma(z) w^{\prime} .
$$

Hence $X_{1}^{V, W}=\mathbb{K} G w^{\prime} \simeq M\left(g z, \sigma_{1}\right)$, and the lemma follows.

Lemma 6.2. Assume that $\rho(z) \sigma(g) \neq 1$. Then $X_{2}^{V, W} \neq 0$. Moreover, $X_{2}^{V, W}$ is absolutely simple if and only if

$$
\rho(g)=-1, \quad(3)_{-\rho(z) \sigma(g)}=0 \quad \text { or } \quad \rho(g z) \sigma(g)=1, \quad(3)_{-\rho(g)}=0 .
$$

In both cases, $X_{2}^{V, W} \simeq M\left(\epsilon g^{2} z, \sigma_{2}\right)$, where $\sigma_{2}$ is the character of $G^{\epsilon}$ with

$$
\sigma_{2}(\epsilon)=\rho(g)(1-\rho(z) \sigma(g)), \quad \sigma_{2}\left(g^{2}\right)=\rho\left(g^{4}\right) \sigma\left(g^{2}\right), \quad \sigma_{2}(z)=\rho\left(z^{2}\right) \sigma(z) .
$$

Let $w^{\prime \prime}=\varphi_{2}\left(\epsilon^{2} v \otimes w^{\prime}\right)$. Then a basis of $X_{2}^{V, W}$ is given by $\left\{w^{\prime \prime}, g w^{\prime \prime}\right\}$. The degrees of these basis vectors are $\epsilon g^{2} z$ and $\epsilon^{2} g^{2} z$ respectively.

Proof. Write $X_{2}^{V, W}=\mathbb{K} G \varphi_{2}\left(v \otimes w^{\prime}\right) \oplus \mathbb{K} G \varphi_{2}\left(\epsilon^{2} v \otimes w^{\prime}\right)$. Then compute

$$
\begin{aligned}
\varphi_{2}\left(v \otimes w^{\prime}\right) & =\left(\mathrm{id}-c_{X_{1}^{V, W}, V} c_{V, X_{1}^{V, W}}\right)\left(v \otimes w^{\prime}\right)+\left(\mathrm{id} \otimes \varphi_{1}\right) c_{1,2}(v \otimes v \otimes w) \\
& =(1+\rho(g))(1-\rho(g z) \sigma(g)) v \otimes w^{\prime}
\end{aligned}
$$

and, using $\varphi_{1}\left(\epsilon^{2} v \otimes w\right)=(1-\rho(z) \sigma(g)) \epsilon^{2} w^{\prime}$,

$$
\begin{aligned}
w^{\prime \prime} & =\varphi_{2}\left(\epsilon^{2} v \otimes w^{\prime}\right) \\
& =\left(\mathrm{id}-c_{X_{1}^{V, W}, V} c_{V, X_{1}^{V, W}}\right)\left(\epsilon^{2} v \otimes w^{\prime}\right)+\left(\mathrm{id} \otimes \varphi_{1}\right) c_{1,2}\left(\epsilon^{2} v \otimes v \otimes w\right) \\
& =\epsilon^{2} v \otimes w^{\prime}-\rho\left(g^{2} z\right) \sigma(g) v \otimes \epsilon w^{\prime}+\rho(g)(1-\rho(z) \sigma(g)) \epsilon v \otimes \epsilon^{2} w^{\prime} .
\end{aligned}
$$

Hence $w^{\prime \prime} \in\left(V \otimes X_{1}^{V, W}\right)_{\epsilon g^{2} z}$ is non-zero. Therefore $w^{\prime \prime}$ is absolutely simple if and only if $(1+\rho(g))(1-\rho(g z) \sigma(g))=0$ and $\epsilon w^{\prime \prime}=\mathbb{K} w^{\prime \prime}$. Since

$$
\epsilon w^{\prime \prime}=v \otimes \epsilon w^{\prime}-\rho\left(g^{2} z\right) \sigma(g) \epsilon v \otimes \epsilon^{2} w^{\prime}+\rho(g)(1-\rho(z) \sigma(g)) \epsilon^{2} v \otimes w^{\prime},
$$

in the case $\rho(g)=-1$ we have $\epsilon w^{\prime \prime}=\mathbb{K} w^{\prime \prime}$ if and only if $(3)_{-\rho(z) \sigma(g)}=0$, and then $\epsilon w^{\prime \prime}=(\rho(z) \sigma(g)-1) w^{\prime \prime}$. Similarly, if $\rho(g z) \sigma(g)=1$, then $\epsilon w^{\prime \prime}=\mathbb{K} w^{\prime \prime}$ if and only if $(3)_{-\rho(g)}=0$, and then $\epsilon w^{\prime \prime}=\rho(g)(1-\rho(z) \sigma(g)) w^{\prime \prime}$. The rest is clear.

Lemma 6.3. Assume that $\rho(z) \sigma(g) \neq 1$ and that $X_{2}^{V, W}$ is absolutely simple. Then $X_{3}^{V, W} \neq 0$ if and only if $\rho(g) \neq-1$. In this case, char $\mathbb{K} \neq 3, \rho(g z) \sigma(g)=1$, $(3)_{-\rho(g)}=0$, the Yetter-Drinfeld module $X_{3}^{V, W}$ is absolutely simple, and $X_{3}^{V, W} \simeq$ $M\left(g^{3} z, \sigma_{3}\right)$, where

$$
\sigma_{3}(g)=\sigma(g), \quad \sigma_{3}(z)=\rho(z)^{3} \sigma(z)
$$


Proof. Acting with $\epsilon g$ on $w^{\prime \prime}=\varphi_{2}\left(\epsilon^{2} v \otimes w^{\prime}\right)$ we obtain that

$$
\varphi_{2}\left(\epsilon^{2} v \otimes \epsilon w^{\prime}\right)=\sigma(g)^{-1}(1-\rho(z) \sigma(g))^{2} g w^{\prime \prime} .
$$

Further, $\varphi_{2}\left(\epsilon^{2} v \otimes \epsilon^{2} w^{\prime}\right)=\epsilon^{2} \varphi_{2}\left(v \otimes w^{\prime}\right)=0$. A direct calculation using these formulas and the expression for $w^{\prime \prime}$ yields

$$
\varphi_{3}\left(\epsilon^{2} v \otimes w^{\prime \prime}\right)=(1+\rho(g))\left(\epsilon^{2} v \otimes w^{\prime \prime}-\rho\left(g^{3} z\right)(1-\rho(z) \sigma(g))^{2} \epsilon v \otimes g w^{\prime \prime}\right) .
$$

Thus $X_{3}^{V, W}=0$ if and only if $\rho(g)=-1$. Assume now that $\rho(g) \neq-1$. Since $X_{2}^{V, W}$ is absolutely simple, Lemma 6.2 implies that $\rho(g z) \sigma(g)=1$ and $(3)_{-\rho(g)}=0$. Then char $\mathbb{K} \neq 3$, since otherwise $(1+\rho(g))^{2}=0$, a contradiction to $\rho(g) \neq-1$. Let $w^{\prime \prime \prime}=(1+\rho(g))^{-1} \varphi_{3}\left(\epsilon^{2} v \otimes w^{\prime \prime}\right)$. Then

$$
w^{\prime \prime \prime}=\epsilon^{2} v \otimes w^{\prime \prime}+\rho\left(g^{2} z\right) \epsilon v \otimes g w^{\prime \prime},
$$

and hence $w^{\prime \prime \prime} \in\left(V \otimes X_{2}^{V, W}\right)_{g^{3} z}$ is non-zero, $X_{3}^{V, W}=\mathbb{K} G w^{\prime \prime \prime}, g w^{\prime \prime \prime}=\sigma(g) w^{\prime \prime \prime}$, and $z w^{\prime \prime \prime}=\rho(z)^{3} \sigma(z) w^{\prime \prime \prime}$. Therefore $X_{3}^{V, W} \simeq M\left(g^{3} z, \sigma_{3}\right)$, where $\sigma_{3}$ is the character of $G^{g}$ with $\sigma_{3}(g)=\sigma(g)$ and $\sigma_{3}(z)=\rho(z)^{3} \sigma(z)$.

Lemma 6.4. Assume that char $\mathbb{K} \neq 3, \rho(g z) \sigma(g)=1$, and $(3)_{-\rho(g)}=0$. Then $X_{4}^{V, W} \neq 0$, and $X_{4}^{V, W}$ is absolutely simple if and only if char $\mathbb{K}=2$. In this case, $X_{4}^{V, W} \simeq M\left(g^{4} z, \sigma_{4}\right)$, where $\sigma_{4}$ is the character of $G$ given by

$$
\sigma_{4}(g)=\rho(g) \sigma(g), \quad \sigma_{4}(\epsilon)=1, \quad \sigma_{4}(z)=\rho(z)^{4} \sigma(z),
$$

and $X_{5}^{V, W}=0$.

Proof. The assumptions imply that $\rho(z) \sigma(g)=\rho(g)^{-1} \neq 1$, and $\rho(g) \neq-1$ since char $\mathbb{K} \neq 3$. Therefore $X_{n}^{V, W}$ is absolutely simple for all $n \in\{1,2,3\}$ by Lemmas 6.1, 6.2, and 6.3. By looking at the support of $V \otimes X_{3}^{V, W}$ we also know that $X_{4}^{V, W}=$ $\mathbb{K} G \varphi_{4}\left(\epsilon^{2} v \otimes w^{\prime \prime \prime}\right) \oplus \mathbb{K} G \varphi_{4}\left(v \otimes w^{\prime \prime \prime}\right)$.

We first obtain that

$$
\begin{aligned}
\varphi_{4}\left(v \otimes w^{\prime \prime \prime}\right)=v \otimes w^{\prime \prime \prime}-c_{X_{3}, V} c_{V, X_{3}}\left(v \otimes w^{\prime \prime \prime}\right)+\rho(g) \epsilon v & \otimes \varphi_{3}\left(v \otimes w^{\prime \prime}\right) \\
& +\rho\left(g^{3} z\right) \epsilon^{2} v \otimes \varphi_{3}\left(v \otimes g w^{\prime \prime}\right) .
\end{aligned}
$$

Now act with $\epsilon$ and $g \epsilon$ on

$$
\varphi_{3}\left(\epsilon^{2} v \otimes w^{\prime \prime}\right)=(1+\rho(g)) w^{\prime \prime \prime}
$$

to obtain that

$$
\begin{aligned}
& \varphi_{3}\left(v \otimes w^{\prime \prime}\right)=\rho(g)^{-2}(1+\rho(g)) \epsilon w^{\prime \prime \prime}, \\
& \varphi_{3}\left(v \otimes g w^{\prime \prime}\right)=\rho(g)^{-3}(1+\rho(g)) \sigma(g) \epsilon^{2} w^{\prime \prime \prime},
\end{aligned}
$$

respectively. Then

$$
\varphi_{4}\left(v \otimes w^{\prime \prime \prime}\right)=\left(1+\rho(g)^{-1}\right)\left(v \otimes w^{\prime \prime \prime}+\epsilon v \otimes \epsilon w^{\prime \prime \prime}+\epsilon^{2} v \otimes \epsilon^{2} w^{\prime \prime \prime}\right)
$$


and hence $\varphi_{4}\left(v \otimes w^{\prime \prime \prime}\right) \in\left(V \otimes X_{3}^{V, W}\right)_{g^{4} z}$ is non-zero.

Act with $\epsilon g$ on Equation (6.1) and use that $\sigma_{2}(\epsilon)=\rho\left(g^{2}\right)$. Thus

$$
\varphi_{3}\left(\epsilon^{2} v \otimes g w^{\prime \prime}\right)=(1+\rho(g)) \rho(g) \sigma(g) \epsilon w^{\prime \prime \prime} .
$$

Now compute

$$
\begin{aligned}
\varphi_{4}\left(\epsilon^{2} v \otimes w^{\prime \prime \prime}\right)= & \epsilon^{2} v \otimes w^{\prime \prime \prime}-\rho\left(g^{2}\right) v \otimes \epsilon w^{\prime \prime \prime} \\
& +\rho(g) \epsilon^{2} v \otimes \varphi_{3}\left(\epsilon^{2} v \otimes w^{\prime \prime}\right)-\rho(z) v \otimes \varphi_{3}\left(\epsilon^{2} v \otimes g w^{\prime \prime}\right) \\
= & \left(1+\rho(g)+\rho(g)^{2}\right)\left(\epsilon^{2} v \otimes w^{\prime \prime \prime}-v \otimes \epsilon w^{\prime \prime \prime}\right) .
\end{aligned}
$$

Thus $X_{4}^{V, W}$ is absolutely simple if and only if $(3)_{\rho(g)}=0$ (in which case char $\mathbb{K}=2$ ) and $\mathbb{K} G \varphi_{4}\left(v \otimes w^{\prime \prime \prime}\right)$ is absolutely simple. Let

$$
w^{\prime \prime \prime \prime}=v \otimes w^{\prime \prime \prime}+\epsilon v \otimes \epsilon w^{\prime \prime \prime}+\epsilon^{2} v \otimes \epsilon^{2} w^{\prime \prime \prime} .
$$

Then $\varphi_{4}\left(v \otimes w^{\prime \prime \prime}\right)=\left(1+\rho(g)^{-1}\right) w^{\prime \prime \prime \prime}$ by Equation (6.2), $g w^{\prime \prime \prime \prime \prime}=\rho(g) \sigma(g) w^{\prime \prime \prime \prime}$, and hence $X_{4}^{V, W} \simeq M\left(g^{4} z, \sigma_{4}\right)$, where $\sigma_{4}$ is the character of $G$ given by $\sigma_{4}(\epsilon)=1$, $\sigma_{4}(g)=\rho(g) \sigma(g)$, and $\sigma_{4}(z)=\rho(z)^{4} \sigma(z)$.

Now we prove that $X_{5}^{V, W}=0$. Observe that

$$
X_{5}^{V, W}=\varphi_{5}\left(V \otimes X_{4}^{V, W}\right)=\mathbb{K} G \varphi_{5}\left(v \otimes w^{\prime \prime \prime \prime}\right) .
$$

By acting with $\epsilon$ and $g \epsilon$ on $\varphi_{4}\left(\epsilon^{2} v \otimes w^{\prime \prime \prime}\right)=0$ we obtain that $\varphi_{4}\left(v \otimes \epsilon w^{\prime \prime \prime}\right)=0$ and $\varphi_{4}\left(v \otimes \epsilon^{2} w^{\prime \prime \prime}\right)=0$, respectively. A direct calculation yields that

$$
\varphi_{5}\left(v \otimes w^{\prime \prime \prime \prime}\right)=(1+\rho(g)) v \otimes w^{\prime \prime \prime \prime}+\rho(g) v \otimes \varphi_{4}\left(v \otimes w^{\prime \prime \prime}\right)=0,
$$

which proves the claim.

Now we compute the modules $(\operatorname{ad} W)^{m}(V)$ for $m \geq 1$. As before, we write $\varphi_{n}=$ $\varphi_{n}^{W, V}$

Lemma 6.5. Let $y_{0}=v$ and let $y_{n}=w \otimes y_{n-1}$ for all $n \geq 1$. Then $y_{n} \in\left(W^{\otimes n} \otimes V\right)_{g z^{n}}$ and $\mathbb{K} G y_{n} \simeq M\left(g z^{n}, \rho_{n}\right)$, where $\rho_{n}$ is the character of $G^{g}$ given by

$$
\rho_{n}(g)=\rho(g) \sigma(g)^{n}, \quad \rho_{n}(z)=\rho(z) \sigma(z)^{n},
$$

and $\varphi_{n}\left(w \otimes y_{n-1}\right)=\gamma_{n} y_{n}$ for all $n \in \mathbb{N}$, where

$$
\gamma_{n}=(n)_{\sigma(z)}\left(1-\rho(z) \sigma\left(g z^{n-1}\right)\right)
$$

Moreover,

$$
X_{n}^{W, V} \simeq \mathbb{K} G\left(\left(\gamma_{1} \cdots \gamma_{n}\right) y_{n}\right)
$$

for all $n \in \mathbb{N}_{0}$.

Proof. We prove by induction on $n$ that $\varphi_{n}\left(w \otimes y_{n-1}\right)=\gamma_{n} y_{n}$ for all $n \geq 1$. The remaining claims are then easily shown. 
It is clear that $\varphi_{1}(w \otimes v)=w \otimes v-g w \otimes z v=(1-\rho(z) \sigma(g)) y_{1}$. Let now $n \geq 1$. Then

$$
\varphi_{n+1}\left(w \otimes y_{n}\right)=w \otimes y_{n}-g z^{n} w \otimes z y_{n}+\sigma(z) w \otimes \varphi_{n}\left(w \otimes y_{n-1}\right),
$$

and hence induction hypothesis implies that

$$
\varphi_{n+1}\left(w \otimes y_{n}\right)=(n+1)_{\sigma(z)}\left(1-\rho(z) \sigma\left(g z^{n}\right)\right) y_{n+1} .
$$

This proves the claimed formula.

Proposition 6.6. Let $V, W \in{ }_{G}^{G} \mathcal{Y D}$ such that $V \simeq M(g, \rho)$, where $\rho$ is a character of $G^{g}$, and $W \simeq M(z, \sigma)$, where $\sigma$ is a character of $G$. Then the following hold:

(1) $(\operatorname{ad} V)^{m}(W)$ and $(\operatorname{ad} W)^{m}(V)$ are absolutely simple or zero for all $m \in \mathbb{N}_{0}$ if and only if

(a) $\rho(z) \sigma(g)=1$, or

(b) $\rho(g)=-1$ and $(3)_{-\rho(z) \sigma(g)}=0$, or

(c) $\rho(g z) \sigma(g)=1,(3)_{\rho(g)}=0$, and char $\mathbb{K}=2$.

(2) Assume that the equations in one of (1a), (1b), (1c) hold. Then the Cartan matrix of the pair $(V, W)$ satisfies

$$
a_{1,2}^{(V, W)}= \begin{cases}0 & \text { in the case (1a) } \\ -2 & \text { in the case (1b) } \\ -4 & \text { in the case (1c) }\end{cases}
$$

Moreover,

(a) if $a_{1,2}^{(V, W)}=-2$ then $X_{2}^{V, W} \simeq M\left(\epsilon g^{2} z, \sigma_{2}\right)$, where $\sigma_{2}$ is the character of $G^{\epsilon}$ given by $\sigma_{2}(\epsilon)=-\rho(z)^{-1} \sigma(g)^{-1}, \sigma_{2}(z)=\rho(z)^{2} \sigma(z)$ and $\sigma_{2}\left(g^{2}\right)=\sigma\left(g^{2}\right)$, (b) if $a_{1,2}^{(V, W)}=-4$ then $X_{4}^{V, W} \simeq M\left(g^{4} z, \sigma_{4}\right)$, where $\sigma_{4}$ is the character of $G$ given by $\sigma_{4}(g)=\rho(g) \sigma(g), \sigma_{4}(\epsilon)=1$, and $\sigma_{4}(z)=\rho(z)^{4} \sigma(z)$.

(3) Assume that the equations in one of (1a), (1b), (1c) hold and let $m \in \mathbb{N}_{0}$. Then $a_{2,1}^{(V, W)}=-m$ if and only if $\gamma_{m+1}=0$ and $\gamma_{1} \cdots \gamma_{m} \neq 0$, where

$$
\gamma_{k}=(k)_{\sigma(z)}\left(1-\rho(z) \sigma\left(g z^{k-1}\right)\right)
$$

for all $k \in \mathbb{N}_{0}$. In this case, $X_{m}^{W, V} \simeq M\left(g z^{m}, \rho_{m}\right)$, where $\rho_{m}$ is the character of $G^{g}$ given by

$$
\rho_{m}(g)=\rho(g) \sigma(g)^{m}, \quad \rho_{m}(z)=\rho(z) \sigma(z)^{m} .
$$

Proof. By Lemmas 6.1, 6.2, 6.3, and 6.4, $(\operatorname{ad} V)^{m}(W)$ is absolutely simple or zero for all $m \in \mathbb{N}$ if and only if $\rho(z) \sigma(g)=1$ or $\rho(g)=-1,(3)_{-\rho(z) \sigma(g)}=0$, or $\rho(g z) \sigma(g)=1$, $(3)_{-\rho(g)}=0$, char $\mathbb{K} \neq 3$, char $\mathbb{K}=2$. By Lemma 6.5 the Yetter-Drinfeld modules $(\operatorname{ad} W)^{m}(V)$ for $m \geq 0$ are absolutely simple or zero. This proves (1). Then (2) is easy to get from the same lemmas. 
Corollary 6.7. Let $V, W \in G_{G}^{G} \mathcal{Y D}$ such that $V \simeq M(g, \rho)$, where $\rho$ is a character of $G^{g}$, and $W \simeq M(z, \sigma)$, where $\sigma$ is a character of $G$, and

$$
\rho(g)=-1, \quad \rho(z) \sigma(g z)=1, \quad 1-\rho(z) \sigma(g)+\rho\left(z^{2}\right) \sigma\left(g^{2}\right)=0 .
$$

Further, let $g^{\prime}=g^{-1}, \epsilon^{\prime}=\epsilon, z^{\prime}=g^{2} z, \rho^{\prime}$ be the character of $G^{g}$ dual to $\rho$, and $\sigma^{\prime}$ the character of $G^{\epsilon}$ given by $\sigma^{\prime}(\epsilon)=-\rho(z)^{-1} \sigma(g)^{-1}, \sigma^{\prime}(z)=\rho(z)^{2} \sigma(z), \sigma^{\prime}\left(g^{2}\right)=\sigma\left(g^{2}\right)$. Then $a_{1,2}^{(V, W)}=-2$ and

$$
R_{1}(V, W)=\left(V^{*}, X_{2}^{V, W}\right)
$$

with $V^{*} \simeq M\left(g^{\prime}, \rho^{\prime}\right), X_{2}^{V, W} \simeq M\left(\epsilon^{\prime} z^{\prime}, \sigma^{\prime}\right)$, and

$$
\rho^{\prime}\left(g^{\prime}\right)=\sigma^{\prime}\left(\epsilon^{\prime} z^{\prime}\right)=-1, \quad \rho^{\prime}\left(z^{\prime}\right)^{2} \sigma^{\prime}\left(\epsilon^{\prime} g^{\prime 2}\right)=1, \quad 1+\sigma^{\prime}\left(\epsilon^{\prime}\right)+\sigma^{\prime}\left(\epsilon^{\prime}\right)^{2}=0 .
$$

Proof. Using Proposition 6.6 one obtains that $a_{1,2}^{(V, W)}=-2$. The rest of the proof is similar to the proof of Corollary 4.13 .

Corollary 6.8. Let $V, W \in G_{G}^{G} \mathcal{Y D}$ such that $V \simeq M(g, \rho)$, where $\rho$ is a character of $G^{g}$, and $W \simeq M(z, \sigma)$, where $\sigma$ is a character of $G$, and

$$
\rho(g)=-1, \quad \rho(z) \sigma(g z)=1, \quad 1-\rho(z) \sigma(g)+\rho\left(z^{2}\right) \sigma\left(g^{2}\right)=0 .
$$

Let $g^{\prime \prime}=g z . \epsilon^{\prime \prime}=\epsilon^{-1}, z^{\prime \prime}=z^{-1}$, $\rho^{\prime \prime}$ be the character of $G^{g}$ given by $\rho^{\prime \prime}(g)=-\sigma(g)$, $\rho^{\prime \prime}(z)=\rho(z) \sigma(z)$, and $\sigma^{\prime \prime}$ be the character of $G$ dual to $\sigma$. Then $a_{2,1}^{(V, W)}=-1$ and

$$
R_{2}(V, W)=\left(X_{1}^{W, V}, W^{*}\right)
$$

with $X_{1}^{W, V} \simeq M\left(g^{\prime \prime}, \rho^{\prime \prime}\right), W^{*} \simeq M\left(z^{\prime \prime}, \sigma^{\prime \prime}\right)$, and

$$
\rho^{\prime \prime}\left(g^{\prime \prime}\right)=-1, \quad \rho^{\prime \prime}\left(z^{\prime \prime}\right) \sigma^{\prime \prime}\left(g^{\prime \prime} z^{\prime \prime}\right)=1, \quad(3)_{-\rho^{\prime \prime}\left(z^{\prime \prime}\right) \sigma^{\prime \prime}\left(g^{\prime \prime}\right)}=0
$$

Proof. Since $\rho(z) \sigma(g) \neq 1$, Proposition 6.6 implies that $a_{2,1}^{(V, W)}=-1$. The rest of the proof is similar to the proof of Corollary 4.14.

\section{Computing the Reflections}

7.1. Pairs of Yetter-Drinfeld modules. Let $G$ be a non-abelian epimorphic image of $\Gamma_{3}$. We first identify pairs $(V, W)$ of Yetter-Drinfeld modules over $G$ with a Cartan matrix of finite type. More precisely, we define classes $\wp_{i}$, where $1 \leq i \leq 6$, of pairs of absolutely simple Yetter-Drinfeld modules over $G$, such that all pairs in these classes can be treated simultaneously with respect to reflections.

Definition 7.1. (The classes $\wp_{i}$ for $1 \leq i \leq 6$ of pairs of Yetter-Drinfeld modules) Let $V$ and $W$ be Yetter-Drinfeld modules over $G$ and let $i \in \mathbb{N}$ with $1 \leq i \leq 6$. We say that $(V, W) \in \wp_{i}$, if there exist $g, \epsilon, z \in G$ such that the following hold.

(1) There is a group epimorphism

$$
\Gamma_{3} \rightarrow G, \quad \gamma \mapsto g, \quad \nu \mapsto \epsilon, \quad \zeta \mapsto z
$$


(2) $V \simeq M(g, \rho)$ for some character $\rho$ of $G$, and either $W \simeq M(\epsilon z, \sigma)$ for some character $\sigma$ of $G^{\epsilon}$ or $W \simeq M(z, \sigma)$ for some absolutely irreducible representation $\sigma$ of $G$.

(3) $W, \rho, \sigma$, and $\mathbb{K}$ satisfy the conditions in the $i$-th row of Table 2 ,

TABle 2. Conditions on the classes $\wp_{i}, 1 \leq i \leq 6$

\begin{tabular}{|c|c|c|c|}
\hline & {$[W]$} & Conditions on $\rho$ and $\sigma$ & char $\mathbb{K}$ \\
\hline$\wp_{1}$ & $M(\epsilon z, \sigma)$ & $\begin{array}{c}\rho(g)=\sigma(\epsilon z)=-1 \\
\rho\left(z^{2}\right) \sigma\left(\epsilon g^{2}\right)=1,(3)_{\sigma(\epsilon)}=0\end{array}$ & \\
\hline$\wp_{2}$ & $M(\epsilon z, \sigma)$ & $\begin{array}{c}\rho(g)=\sigma(z)=-1 \\
\rho\left(z^{2}\right) \sigma\left(\epsilon g^{2}\right)=\sigma(\epsilon)=1\end{array}$ & $\neq 3$ \\
\hline$\wp_{3}$ & $M(z, \sigma)$ & $\begin{array}{c}\rho(g)=\sigma(z)=-1 \\
\rho\left(z^{2}\right) \sigma\left(g^{2}\right)=1, \operatorname{deg} \sigma=2\end{array}$ & $\neq 3$ \\
\hline$\wp_{4}$ & $M(z, \sigma)$ & $\begin{array}{c}\rho(g)=-1, \rho(z) \sigma(g z)=1 \\
(3)_{-\rho(z) \sigma(g)}=0, \operatorname{deg} \sigma=1\end{array}$ & \\
\hline$\wp_{5}$ & $M(z, \sigma)$ & $\begin{array}{c}\rho(g)=\sigma(z)=1,(3)_{\rho(z) \sigma(g)}=0 \\
\operatorname{deg} \sigma=1\end{array}$ & 2 \\
\hline$\wp_{6}$ & $M(z, \sigma)$ & $\rho(g)=\sigma(z)=-1,(3)_{-\rho(z) \sigma(g)}=0$ & $\neq 2,3$ \\
\hline
\end{tabular}

Proposition 7.2. Let $V, W$ be absolutely simple Yetter-Drinfeld modules over $G$. Suppose that $c_{W, V} c_{V, W} \neq \operatorname{id}_{V \otimes W}$, the pair $(V, W)$ admits all reflections, the Weyl groupoid of $(V, W)$ is finite, and $|\operatorname{supp} V| \geq|\operatorname{supp} W|$. Then the Cartan matrix of $(V, W)$ is of finite type if and only if $(V, W)$ belongs to one of the classes $\wp_{i}$ for $1 \leq i \leq 5$ in Table 2. In this case $a_{1,2}^{(V, W)}=-2$ and $a_{2,1}^{(V, W)}=-1$.

Proof. From [25, Thm. 7.3] we know that the quandle $\operatorname{supp}(V \oplus W)$ is isomorphic to $Z_{3}^{3,1}$ or $Z_{3}^{3,2}$. Since $|\operatorname{supp} V| \geq|\operatorname{supp} W|$, we conclude that $|\operatorname{supp} V|=3$ and $|\operatorname{supp} W| \leq 2$. Let $g, g^{\prime} \in \operatorname{supp} V$ with $g^{\prime} \neq g$ and let $\epsilon=g^{\prime-1} g$. Then $\epsilon \neq 1$ and $\epsilon^{3}=1$ by (3.1). Since $V$ is absolutely simple, there exists a character $\rho$ of $G$ such that $V \simeq M(g, \rho)$. Since $W$ is absolutely simple and $\operatorname{supp}(V \oplus W)$ generates $G$, there exists $z \in Z(G)$ such that either $W \simeq M(\epsilon z, \sigma)$ for a character $\sigma$ of $G^{\epsilon}$, or $W \simeq M(z, \sigma)$ for some absolutely irreducible representation $\sigma$ of $G$. Then $\operatorname{deg} \sigma \leq 2$ by Lemma 3.2 .

Since the pair $(V, W)$ admits all reflections and the Weyl groupoid of $(V, W)$ is finite, we conclude from [22, Theorem 2.5] that $(\operatorname{ad} V)^{m}(W)$ and $(\operatorname{ad} W)^{m}(V)$ are absolutely simple or zero for all $m \geq 1$. The condition $c_{W, V} c_{V, W} \neq \mathrm{id}_{V \otimes W}$ just means that the Cartan matrix of $(V, W)$ is not of type $A_{1} \times A_{1}$. Propositions 4.12, 5.9, and 6.6 imply that the Cartan matrix of $(V, W)$ is of finite type if and only if $(V, W)$ belongs to one of the classes $\wp_{i}$ for $1 \leq i \leq 6$ in Table 2. Moreover, in this case $a_{1,2}^{(V, W)}=-2$ and $a_{2,1}^{(V, W)}=-1$. 
We have to exclude the class $\wp_{6}$. To do so, it suffices to prove that if $(V, W) \in \wp_{6}$ then not all assumptions of the proposition are fulfilled.

Assume that $(V, W)$ belongs to $\wp_{6}$. By Proposition 6.6.

$$
R_{2}(V, W)=\left((\operatorname{ad} W)(V), W^{*}\right) \simeq\left(M\left(g z, \rho_{1}\right), M\left(z^{-1}, \sigma^{-1}\right)\right),
$$

where $\rho_{1}$ is the character of $G^{g}$ with $\rho_{1}(g)=\rho(g) \sigma(g)$ and $\rho_{1}(z)=\rho(z) \sigma(z)$. Let $\left(V^{\prime}, W^{\prime}\right)=R_{2}(V, W)$. We obtain that $\rho_{1}(g z)=\rho(z) \sigma(g) \notin\{1,-1\}$, since $(3)_{-\rho(z) \sigma(g)}=0$ and char $\mathbb{K} \neq 3$. Since also char $\mathbb{K} \neq 2$ by assumption on $\wp_{6}$, Proposition 6.6 implies that not all of $\left(\operatorname{ad} V^{\prime}\right)^{m}\left(W^{\prime}\right)$ and $\left(\operatorname{ad} W^{\prime}\right)^{m}\left(V^{\prime}\right)$ for $m \geq 1$ are absolutely simple or zero. This is a contradiction to the assumption that $(V, W)$ admits all reflections and the Weyl groupoid of $(V, W)$ is finite.

Let $(V, W)$ be a pair of Yetter-Drinfeld modules over $G$. If $(V, W) \in \wp_{1}$, then $R_{1}(V, W) \in \wp_{4}$ and $R_{2}(V, W) \in \wp_{1}$ by Corollaries 4.13 and 4.14. On the other hand, if $(V, W) \in \wp_{4}$, then $R_{1}(V, W) \in \wp_{1}$ and $R_{2}(V, W) \in \wp_{4}$ by Corollaries 6.7 and 6.8. We display this fact in the following graph:

$$
\wp_{1} \frac{R_{1}}{\wp_{4}}
$$

We omit $R_{2}$ in the graph, since it fixes the classes $\wp_{1}$ and $\wp_{4}$. Since the Cartan matrices of $(V, W)$ are the same for all reflections of $(V, W)$ in the classes $\wp_{1}$ and $\wp_{4}$, we conclude the following.

Lemma 7.3. Let $(V, W)$ be a pair in $\wp_{1}$ or $\wp_{4}$. Then $(V, W)$ admits all reflections and the Weyl groupoid of $(V, W)$ is standard of type $B_{2}$.

Similarly to the previous paragraph, Corollaries 4.15, 4.16, 5.10, and 5.11 imply that the reflections of the pairs $(V, W)$ in $\wp_{2}$ and $\wp_{3}$ can be displayed in the following graph:

$$
\wp_{2} \stackrel{R_{1}}{\wp_{3}}
$$

Lemma 7.4. Let $(V, W)$ be a pair in $\wp_{2}$ or $\wp_{3}$. Then $(V, W)$ admits all reflections and the Weyl groupoid of $(V, W)$ is standard of type $B_{2}$.

The reflections of the pairs $(V, W) \in \wp_{5}$ show a more complicated pattern.

Let us assume that char $\mathbb{K}=2$. Lemmas 7.5, 7.8, 7.6, 7.9, 7.7, and 7.10 below imply that the reflections of the pairs $(V, W)$ in $\wp_{5}$ can be displayed in the following graph:

$$
\wp_{5}^{\prime} \stackrel{R_{1}}{\wp_{5}} \stackrel{R_{2}}{ } \wp_{5}^{\prime \prime}
$$

The classes $\wp_{5}^{\prime}$ and $\wp_{5}^{\prime \prime}$ are defined in the same way as $\wp_{5}$ in Definition 7.1 , except that in the last line one refers to the conditions in Table 3 . Since the class $\wp_{5}$ is 
non-empty only if char $\mathbb{K}=2$, in the definitions of $\wp_{5}^{\prime}$ and $\wp_{5}^{\prime \prime}$ we also assume that $\operatorname{char} \mathbb{K}=2$.

TABLE 3. Conditions on the classes $\wp_{5}, \wp_{5}^{\prime}, \wp_{5}^{\prime \prime}$

\begin{tabular}{|c|c|c|}
\hline & {$[W]$} & Conditions on $\rho$ and $\sigma$ \\
\hline$\wp_{5}$ & $M(z, \sigma)$ & $\rho(g)=\sigma(z)=1,(3)_{\rho(z) \sigma(g)}=0, \operatorname{deg} \sigma=1$ \\
\hline$\wp_{5}^{\prime}$ & $M(\epsilon z, \sigma)$ & $(3)_{\sigma(\epsilon)}=0, \sigma(z)=\sigma(\epsilon), \rho\left(z^{2}\right) \sigma\left(\epsilon g^{2}\right)=1, \rho(g)=1$ \\
\hline$\wp_{5}^{\prime \prime}$ & $M(z, \sigma)$ & $(3)_{\rho(g)}=0, \sigma(z)=1, \rho(g z) \sigma(g)=1, \operatorname{deg} \sigma=1$ \\
\hline
\end{tabular}

Lemma 7.5. Let $(V, W) \in \wp_{5}$ and $g, \epsilon, z, \rho, \sigma$ as in Definition 7.1 such that $V \simeq$ $M(g, \rho)$ and $W \simeq M(z, \sigma)$. Let $g^{\prime}=g^{-1}, \epsilon^{\prime}=\epsilon, z^{\prime}=g^{2} z$, $\rho^{\prime}$ be the character of $G^{g}$ dual to $\rho$, and $\sigma^{\prime}$ be the character of $G^{\epsilon}$ given by $\sigma^{\prime}(\epsilon)=-\rho(z)^{-1} \sigma(g)^{-1}$, $\sigma^{\prime}(z)=\rho(z)^{2}, \sigma^{\prime}\left(g^{2}\right)=\sigma\left(g^{2}\right)$. Then $a_{1,2}^{(V, W)}=-2$ and

$$
R_{1}(V, W)=\left(V^{*}, X_{2}^{V, W}\right)
$$

with $V^{*} \simeq M\left(g^{\prime}, \rho^{\prime}\right), X_{2}^{V, W} \simeq M\left(\epsilon^{\prime} z^{\prime}, \sigma^{\prime}\right)$, and

$$
\rho^{\prime}\left(g^{\prime}\right)=1, \quad \rho^{\prime}\left(z^{\prime 2}\right) \sigma^{\prime}\left(\epsilon^{\prime} g^{\prime 2}\right)=1, \quad \sigma^{\prime}\left(z^{\prime}\right)=\sigma^{\prime}\left(\epsilon^{\prime}\right), \quad(3)_{\sigma^{\prime}\left(\epsilon^{\prime}\right)}=0 .
$$

In particular, $R_{1}(V, W) \in \wp_{5}^{\prime}$.

Proof. Using Proposition 6.6 we obtain that $a_{1,2}^{(V, W)}=-2$ and hence the description of $R_{1}(V, W)$ together with the isomorphisms regarding $V^{*}$ and $X_{2}^{V, W}$ follow from the same Proposition. The remaining claims are easy to check.

Lemma 7.6. Let $(V, W) \in \wp_{5}^{\prime}$ and $g, \epsilon, z, \rho, \sigma$ as in Definition 7.1 such that $V \simeq$ $M(g, \rho)$ and $W \simeq M(\epsilon z, \sigma)$. Let $g^{\prime}=g^{-1}, \epsilon^{\prime}=\epsilon, z^{\prime}=g^{2} z, \rho^{\prime}$ be the character of $G^{g}$ dual to $\rho$, and $\sigma^{\prime}$ be the character of $G$ given by $\sigma^{\prime}(\epsilon)=1, \sigma^{\prime}(g)=\rho(z)^{-1} \sigma(\epsilon)$, $\sigma^{\prime}(z)=\rho(z)^{2} \sigma(z)$. Then $a_{1,2}^{(V, W)}=-2$ and

$$
R_{1}(V, W)=\left(V^{*}, X_{2}^{V, W}\right)
$$

with $V^{*} \simeq M\left(g^{\prime}, \rho^{\prime}\right), X_{2}^{V, W} \simeq M\left(z^{\prime}, \sigma^{\prime}\right)$, and

$$
\rho^{\prime}\left(g^{\prime}\right)=\sigma^{\prime}\left(z^{\prime}\right)=1, \quad 1+\rho^{\prime}\left(z^{\prime}\right) \sigma^{\prime}\left(g^{\prime}\right)+\left(\rho^{\prime}\left(z^{\prime}\right) \sigma^{\prime}\left(g^{\prime}\right)\right)^{2}=0 .
$$

In particular, $R_{1}(V, W) \in \wp_{5}$.

Proof. It is similar to the proof of Lemma 7.5, but one needs Proposition 4.12 ,

Lemma 7.7. Let $(V, W) \in \wp_{5}^{\prime \prime}$ and $g, \epsilon, z, \rho, \sigma$ as in Definition 7.1 such that $V \simeq$ $M(g, \rho)$ and $W \simeq M(z, \sigma)$. Let $g^{\prime}=g^{-1}, \epsilon^{\prime}=\epsilon, z^{\prime}=g^{4} z$, $\rho^{\prime}$ be the character of $G^{g}$ 
dual to $\rho$, and $\sigma^{\prime}$ be the character of $G$ given by $\sigma^{\prime}(g)=\rho(g) \sigma(g), \sigma^{\prime}(z)=\rho(z)^{4} \sigma(z)$, $\sigma^{\prime}(\epsilon)=1$. Then $a_{1,2}^{(V, W)}=-4$ and

$$
R_{1}(V, W)=\left(V^{*}, X_{4}^{V, W}\right)
$$

with $V^{*} \simeq M\left(g^{\prime}, \rho^{\prime}\right), X_{4}^{V, W} \simeq M\left(z^{\prime}, \sigma^{\prime}\right)$, and

$$
\rho^{\prime}\left(g^{\prime} z^{\prime}\right) \sigma^{\prime}\left(g^{\prime}\right)=1, \quad 1+\rho^{\prime}\left(g^{\prime}\right)+\rho^{\prime}\left(g^{\prime}\right)^{2}=0, \quad \sigma^{\prime}\left(z^{\prime}\right)=1 .
$$

In particular, $R_{1}(V, W) \in \wp_{5}^{\prime \prime}$.

Proof. It is similar to the proof of Lemma 7.5 .

Lemma 7.8. Let $(V, W) \in \wp_{5}$ and $g, \epsilon, z, \rho, \sigma$ as in Definition 7.1 such that $V \simeq$ $M(g, \rho)$ and $W \simeq M(z, \sigma)$. Let $g^{\prime \prime}=g z, \epsilon^{\prime \prime}=\epsilon^{-1}, z^{\prime \prime}=z^{-1}, \rho^{\prime \prime}$ be the character of $G^{g}$ given by $\rho^{\prime \prime}(g)=\sigma(g), \rho^{\prime \prime}(z)=\rho(z)$, and $\sigma^{\prime \prime}$ be the character of $G$ dual to $\sigma$. Then $a_{2,1}^{V, W}=-1$ and

$$
R_{2}(V, W)=\left(X_{1}^{W, V}, W^{*}\right)
$$

with $X_{1}^{W, V} \simeq M\left(g^{\prime \prime}, \rho^{\prime \prime}\right), W^{*} \simeq M\left(z^{\prime \prime}, \sigma^{\prime \prime}\right)$, and

$$
\rho^{\prime \prime}\left(g^{\prime \prime} z^{\prime \prime}\right) \sigma^{\prime \prime}\left(g^{\prime \prime}\right)=1, \quad 1+\rho^{\prime \prime}\left(g^{\prime \prime}\right)+\rho^{\prime \prime}\left(g^{\prime \prime}\right)^{2}=0, \quad \sigma^{\prime \prime}\left(z^{\prime \prime}\right)=1 .
$$

In particular, $R_{2}(V, W) \in \wp_{5}^{\prime \prime}$.

Proof. It is similar to the proof of Lemma 7.5 .

Lemma 7.9. Let $(V, W) \in \wp_{5}^{\prime}$ and $g, \epsilon, z, \rho, \sigma$ as in Definition 7.1 such that $V \simeq$ $M(g, \rho)$ and $W \simeq M(\epsilon z, \sigma)$. Let $g^{\prime \prime}=g z^{2}, \epsilon^{\prime \prime}=\epsilon^{-1}, z^{\prime \prime}=z^{-1}$, $\rho^{\prime \prime}$ be the character of $G^{g}$ given by $\rho^{\prime \prime}(g)=\rho(z)^{-2} \sigma(z)^{-1}, \rho^{\prime \prime}(z)=\rho(z) \sigma(z)^{2}$, and $\sigma^{\prime \prime}$ be the character of $G$ dual to $\sigma$. Then $a_{2,1}^{(V, W)}=-2$ and

$$
R_{2}(V, W)=\left(X_{2}^{W, V}, W^{*}\right)
$$

with $X_{2}^{W, V} \simeq M\left(g^{\prime \prime}, \rho^{\prime \prime}\right), W^{*} \simeq M\left(z^{\prime \prime}, \sigma^{\prime \prime}\right)$, and

$$
\rho^{\prime \prime}\left(g^{\prime \prime}\right)=1, \quad \rho^{\prime \prime}\left(z^{\prime \prime 2}\right) \sigma^{\prime \prime}\left(\epsilon^{\prime \prime} g^{\prime \prime 2}\right)=1, \quad \sigma^{\prime \prime}\left(z^{\prime \prime}\right)=\sigma^{\prime \prime}\left(\epsilon^{\prime \prime}\right), \quad(3)_{\sigma^{\prime \prime}\left(\epsilon^{\prime \prime}\right)}=0 .
$$

In particular, $R_{2}(V, W) \in \wp_{5}^{\prime}$.

Proof. It is similar to the proof of Lemma 7.6 .

Lemma 7.10. Let $(V, W) \in \wp_{5}^{\prime \prime}$ and $g, \epsilon, z, \rho, \sigma$ as in Definition 7.1 such that $V \simeq$ $M(g, \rho)$ and $W \simeq M(z, \sigma)$. Let $g^{\prime \prime}=g z, \epsilon^{\prime \prime}=\epsilon^{-1}, z^{\prime \prime}=z^{-1}, \rho^{\prime \prime}$ be the character of $G^{g}$ given by $\rho^{\prime \prime}(g)=\rho(g) \sigma(g), \rho^{\prime \prime}(z)=\rho(z) \sigma(z)$, and $\sigma^{\prime \prime}$ be the character of $G$ dual to $\sigma$. Then $a_{2,1}^{(V, W)}=-1$ and

$$
R_{2}(V, W)=\left(X_{1}^{W, V}, W^{*}\right)
$$


TABle 4. Classes of Yetter-Drinfeld modules

\begin{tabular}{|c|c|c|c|c|c|}
\hline & $x$ & $\tau$ & $\operatorname{char} \mathbb{K}$ & Hilbert series & Ref. \\
\hline $\mathcal{Y}_{1}$ & $g$ & $\tau(g)=-1$ & & $(2)_{t}^{2}(3)_{t}$ & \begin{tabular}{|l|}
29 \\
\end{tabular} \\
\hline $\mathcal{Y}_{2}$ & $g$ & & 2 & ${ }_{t}(6)_{t}(6)_{t^{2}}$ & [21, $\operatorname{Prc}$ \\
\hline $\mathcal{Y}_{3}$ & $z$ & $\tau(z)=-1$ & & & [31, \\
\hline $\mathcal{Y}_{4}$ & $z$ & $\operatorname{deg} \tau=$ & $p \in P$ & $\left(h_{\eta}\right.$ & 31, \\
\hline $\mathcal{Y}_{5}$ & $z$ & & & & [31, \\
\hline $\mathcal{Y}_{6}$ & $\epsilon z$ & $\tau(\epsilon)=0$ & & $(3)_{t}^{2}$ & 31 , \\
\hline $\mathcal{Y}_{7}$ & $\epsilon z$ & $\tau(\epsilon)=1, \tau(z)$ & & $(2)_{t}^{2}$ & [31, § \\
\hline $\mathcal{Y}_{8}$ & $\epsilon z$ & $\tau(\epsilon z)=-1,(3)_{\tau(\epsilon)}=0$ & $p \in P$ & $(2)_{t}\left(h_{p}^{\prime}\right)_{t}$ & [17, Prop. 2.11] \\
\hline
\end{tabular}

with $X_{1}^{W, V} \simeq M\left(g^{\prime \prime}, \rho^{\prime \prime}\right), W^{*} \simeq M\left(z^{\prime \prime}, \sigma^{\prime \prime}\right)$, and

$$
\rho^{\prime \prime}\left(g^{\prime \prime}\right)=\sigma^{\prime \prime}\left(z^{\prime \prime}\right)=1, \quad(3)_{\rho^{\prime \prime}\left(z^{\prime \prime}\right) \sigma^{\prime \prime}\left(g^{\prime \prime}\right)}=0 .
$$

In particular, $R_{2}(V, W) \in \wp_{5}$.

Proof. It is similar to the proof of Lemma 7.5 ,

\section{Nichols Algebras OVer $\Gamma_{3}$}

8.1. Simple Yetter-Drinfeld modules. In this section, let $G$ be a non-abelian epimorphic image of $\Gamma_{3}$ and let $g, \epsilon, z \in G$ such that the group epimorphism $\Gamma_{3} \rightarrow G$ maps $\gamma \mapsto g, \nu \mapsto \epsilon$ and $\zeta \mapsto z$.

Let $P=\{0\} \cup\{p \in \mathbb{N} \mid p$ is prime $\}$. Let $h_{2}=3, h_{3}=2$ and $h_{p}=6$ for all $p \in P \backslash\{2,3\}$, and $h_{3}^{\prime}=2, h_{p}^{\prime}=6$ for all $p \in P \backslash\{3\}$. For all $1 \leq i \leq 8$ let $\mathcal{Y}_{i}$ be the class of Yetter-Drinfeld modules $U$ over $G$ such that there exist $x, g, \epsilon, z \in G$ and an absolutely irreducible representation $\tau$ of $G^{x}$ such that $U \simeq M(x, \tau)$ and $x$, $\tau$, and $\mathbb{K}$ satisfy the conditions in Table 4 . In Table 4 we also provide the Hilbert series of the Nichols algebras of the Yetter-Drinfeld modules in the classes $\mathcal{Y}_{i}$ for all $1 \leq i \leq 8$ and the references to these Hilbert series.

8.2. In this subsection we collect the examples of Nichols algebras related to the graph

$$
\wp_{1} \stackrel{R_{1}}{\wp_{4}}
$$

Theorem 8.1. Let $p=$ char $\mathbb{K}$. Let $V, W \in{ }_{G}^{G} \mathcal{Y D}$ such that $V \simeq M(g, \rho)$, where $\rho$ is a character of $G^{g}$, and $W \simeq M(\epsilon z, \sigma)$, where $\sigma$ is a character of $G^{\epsilon}$. Assume that $(V, W) \in \wp_{1}$, that is,

$$
\rho(g)=-1, \quad \rho\left(z^{2}\right) \sigma\left(\epsilon g^{2}\right)=1, \quad 1+\sigma(\epsilon)+\sigma(\epsilon)^{2}=0, \quad \sigma(\epsilon z)=-1 .
$$

Then $W \in \mathcal{Y}_{8},(\operatorname{ad} V)(W) \in \mathcal{Y}_{1},(\operatorname{ad} V)^{2}(W) \in \mathcal{Y}_{4}, V \in \mathcal{Y}_{1}$, and

$$
\mathfrak{B}(V \oplus W) \simeq \mathfrak{B}(W) \otimes \mathfrak{B}((\operatorname{ad} V)(W)) \otimes \mathfrak{B}\left((\operatorname{ad} V)^{2}(W)\right) \otimes \mathfrak{B}(V)
$$


as $\mathbb{N}_{0}^{2}$-graded vector spaces in $G_{G}^{G} \mathcal{Y} \mathcal{D}$. In particular, the Hilbert series of $\mathfrak{B}(V \oplus W)$ is

$$
\mathcal{H}\left(t_{1}, t_{2}\right)=(2)_{t_{2}}\left(h_{p}^{\prime}\right)_{t_{2}}(2)_{t_{1} t_{2}}^{2}(3)_{t_{1} t_{2}}\left(h_{p}\right)_{t_{1}^{2} t_{2}}(2)_{t_{1}}^{2}(3)_{t_{1}}
$$

and

$$
\operatorname{dim} \mathfrak{B}(V \oplus W)= \begin{cases}10368 & \text { if char } \mathbb{K} \notin\{2,3\} \\ 5184 & \text { if } \operatorname{char} \mathbb{K}=2, \\ 1152 & \text { if char } \mathbb{K}=3 .\end{cases}
$$

Proof. The Cartan scheme of $(V, W)$ is standard of type $B_{2}$ by Lemma 7.3 . The Yetter-Drinfeld modules $(\operatorname{ad} V)(W)$ and $(\operatorname{ad} V)^{2}(W)$ are in the claimed classes because of Lemmas 4.3 and 4.6. Then the claims concerning the decomposition and the Hilbert series of $\mathfrak{B}(V \oplus W)$ follow from [22, Cor. 2.7(2)], [22, Thm. 2.6] and Table 4 .

Theorem 8.2. Let $p=\operatorname{char} \mathbb{K}$. Let $V, W \in{ }_{G}^{G} \mathcal{Y D}$ such that $V \simeq M(g, \rho)$, where $\rho$ is a character of $G^{g}$, and $W \simeq M(z, \sigma)$, where $\sigma$ is a character of $G$. Assume that $(V, W) \in \wp_{4}$, that is,

$$
\rho(g)=-1, \quad \rho(z) \sigma(g z)=1, \quad 1-\rho(z) \sigma(g)+(\rho(z) \sigma(g))^{2}=0 .
$$

Then $W \in \mathcal{Y}_{4},(\operatorname{ad} V)(W) \in \mathcal{Y}_{1},(\operatorname{ad} V)^{2}(W) \in \mathcal{Y}_{8}, V \in \mathcal{Y}_{1}$, and

$$
\mathfrak{B}(V \oplus W) \simeq \mathfrak{B}(W) \otimes \mathfrak{B}((\operatorname{ad} V)(W)) \otimes \mathfrak{B}\left((\operatorname{ad} V)^{2}(W)\right) \otimes \mathfrak{B}(V)
$$

as $\mathbb{N}_{0}^{2}$-graded vector spaces in $G_{G}^{G} \mathcal{Y} \mathcal{D}$. In particular, the Hilbert series of $\mathfrak{B}(V \oplus W)$ is

$$
\mathcal{H}\left(t_{1}, t_{2}\right)=\left(h_{p}\right)_{t_{2}}(2)_{t_{1} t_{2}}^{2}(3)_{t_{1} t_{2}}(2)_{t_{1}^{2} t_{2}}\left(h_{p}^{\prime}\right)_{t_{1}^{2} t_{2}}(2)_{t_{1}}^{2}(3)_{t_{1}}
$$

and

$$
\operatorname{dim} \mathfrak{B}(V \oplus W)= \begin{cases}10368 & \text { if char } \mathbb{K} \notin\{2,3\} \\ 5184 & \text { if char } \mathbb{K}=2, \\ 1152 & \text { if char } \mathbb{K}=3 .\end{cases}
$$

Proof. It is similar to the proof of Theorem 8.1. For the structure of $(\operatorname{ad} V)^{m}(W)$ for $m=1,2$ see Lemmas 6.1 and 6.2. Alternatively, one can deduce the claim from Theorem 8.1 since $R_{1}(V, W) \in \wp_{1}$ and $R_{1}^{2}(V, W) \simeq(V, W)$.

8.3. Now we list the examples of Nichols algebras related to the graph

$$
\wp_{2} \frac{R_{1}}{\wp_{3}}
$$

Theorem 8.3. Let $V, W \in{ }_{G}^{G} \mathcal{Y D}$ such that $V \simeq M(g, \rho)$, where $\rho$ is a character of $G^{g}$, and $W \simeq M(\epsilon z, \sigma)$, where $\sigma$ is a character of $G^{\epsilon}$. Assume that char $\mathbb{K} \neq 3$ and $(V, W) \in \wp_{2}$, that is,

$$
\rho(g)=\sigma(z)=-1, \quad \rho\left(z^{2}\right) \sigma\left(\epsilon g^{2}\right)=\sigma(\epsilon)=1 .
$$


Then $W \in \mathcal{Y}_{7},(\operatorname{ad} V)(W) \in \mathcal{Y}_{1},(\operatorname{ad} V)^{2}(W) \in \mathcal{Y}_{5}, V \in \mathcal{Y}_{1}$, and

$$
\mathfrak{B}(V \oplus W) \simeq \mathfrak{B}(W) \otimes \mathfrak{B}((\operatorname{ad} V)(W)) \otimes \mathfrak{B}\left((\operatorname{ad} V)^{2}(W)\right) \otimes \mathfrak{B}(V)
$$

as $\mathbb{N}_{0}^{2}$-graded vector spaces in ${ }_{G}^{G} \mathcal{Y D}$. In particular, the Hilbert series of $\mathfrak{B}(V \oplus W)$ is

$$
\mathcal{H}\left(t_{1}, t_{2}\right)=(2)_{t_{2}}^{2}(2)_{t_{1} t_{2}}^{2}(3)_{t_{1} t_{2}}(2)_{t_{1}^{2} t_{2}}^{2}(2)_{t_{1}}^{2}(3)_{t_{1}}
$$

and $\operatorname{dim} \mathfrak{B}(V \oplus W)=2^{8} 3^{2}=2304$.

Proof. The Cartan scheme of $(V, W)$ is standard of type $B_{2}$ by Lemma 7.4 and the decomposition of $\mathfrak{B}(V \oplus W)$ follows from [22, Cor. 2.7(2) and Thm. 2.6]. It is clear that $V \in \mathcal{Y}_{1}$ and $W \in \mathcal{Y}_{7}$. Using Lemmas 4.3 and 4.6 we obtain that $(\operatorname{ad} V)(W) \in \mathcal{Y}_{1}$ and $(\operatorname{ad} V)^{2}(W) \in \mathcal{Y}_{5}$. Now a direct calculation using Table 4 yields the Hilbert series of $\mathfrak{B}(V \oplus W)$.

Theorem 8.4. Let $V, W \in{ }_{G}^{G} \mathcal{Y D}$ such that $V \simeq M(g, \rho)$, where $\rho$ is a character of $G^{g}$, and $W \simeq M(z, \sigma)$, where $\sigma$ is an absolutely irreducible representation of $G$ of degree two. Assume that char $\mathbb{K} \neq 3$ and $(V, W) \in \wp_{3}$, that is,

$$
\rho(g)=\sigma(z)=-1, \quad \rho\left(z^{2}\right) \sigma\left(g^{2}\right)=1 .
$$

Then $W \in \mathcal{Y}_{5},(\operatorname{ad} V)(W) \in \mathcal{Y}_{1},(\operatorname{ad} V)^{2}(W) \in \mathcal{Y}_{7}, V \in \mathcal{Y}_{1}$, and

$$
\mathfrak{B}(V \oplus W) \simeq \mathfrak{B}(W) \otimes \mathfrak{B}((\operatorname{ad} V)(W)) \otimes \mathfrak{B}\left((\operatorname{ad} V)^{2}(W)\right) \otimes \mathfrak{B}(V)
$$

as $\mathbb{N}_{0}^{2}$-graded vector spaces in ${ }_{G}^{G} \mathcal{Y D}$. In particular, the Hilbert series of $\mathfrak{B}(V \oplus W)$ is

$$
\mathcal{H}\left(t_{1}, t_{2}\right)=(2)_{t_{2}}^{2}(2)_{t_{1} t_{2}}^{2}(3)_{t_{1} t_{2}}(2)_{t_{1}^{2} t_{2}}^{2}(2)_{t_{1}}^{2}(3)_{t_{1}}
$$

and $\operatorname{dim} \mathfrak{B}(V \oplus W)=2^{8} 3^{2}=2304$.

Proof. It is similar to the proof of Theorem 8.3 . Here one has to use Lemmas 5.2 and 5.4. Alternatively, one can deduce the claim from Theorem 8.3 since $R_{1}(V, W) \in \wp_{2}$ and $R_{1}^{2}(V, W) \simeq(V, W)$.

8.4. Now we list the examples related to the following graph:

$$
\wp_{5}^{\prime} \frac{R_{1}}{\wp_{5}} \frac{R_{2}}{\wp_{5}^{\prime \prime}}
$$

Lemma 8.5. Let $I=\{1,2\}, \mathcal{X}=\{a, b, c\}, r_{1}=(a b) \in \mathbb{S}_{\mathcal{X}}, r_{2}=(b c) \in \mathbb{S}_{\mathcal{X}}$,

$$
A^{a}=\left(\begin{array}{cc}
2 & -2 \\
-2 & 2
\end{array}\right), \quad A^{b}=\left(\begin{array}{cc}
2 & -2 \\
-1 & 2
\end{array}\right), \quad A^{c}=\left(\begin{array}{cc}
2 & -4 \\
-1 & 2
\end{array}\right) \text {. }
$$

Then the following hold:

(1) $\mathcal{C}=\mathcal{C}\left(I, \mathcal{X},\left(r_{i}\right)_{i \in I},\left(A^{X}\right)_{X \in \mathcal{X}}\right)$ is a Cartan scheme. 
(2) $\left(\Delta^{\mathrm{reX}}\right)_{X \in \mathcal{X}}$ forms a finite irreducible root system of type $\mathcal{C}$, and

$$
\begin{aligned}
& \Delta_{+}^{\mathrm{re} a}=\left\{\alpha_{1}, \alpha_{2}, \alpha_{1}+\alpha_{2}, 2 \alpha_{1}+\alpha_{2}, \alpha_{1}+2 \alpha_{2}, 2 \alpha_{1}+3 \alpha_{2}\right\}, \\
& \Delta_{+}^{\mathrm{re} b}=\left\{\alpha_{1}, \alpha_{2}, \alpha_{1}+\alpha_{2}, 2 \alpha_{1}+\alpha_{2}, 3 \alpha_{1}+2 \alpha_{2}, 4 \alpha_{1}+3 \alpha_{2}\right\}, \\
& \Delta_{+}^{\mathrm{re} c}=\left\{\alpha_{1}, \alpha_{2}, \alpha_{1}+\alpha_{2}, 2 \alpha_{1}+\alpha_{2}, 3 \alpha_{1}+\alpha_{2}, 4 \alpha_{1}+\alpha_{2}\right\} .
\end{aligned}
$$

Proof. Both claims follow from the definitions. The Cartan scheme $\mathcal{C}$ appeared already in [13, Thm. 6.1].

Theorem 8.6. Let $V, W \in{ }_{G}^{G} \mathcal{Y D}$ such that $V \simeq M(g, \rho)$, where $\rho$ is a character of $G^{g}$, and $W \simeq M(z, \sigma)$, where $\sigma$ is a character of $G$. Assume that char $\mathbb{K}=2$ and $(V, W) \in \wp_{5}$, that is,

$$
\rho(g)=\sigma(z)=1, \quad 1+\rho(z) \sigma(g)+\rho(z)^{2} \sigma(g)^{2}=0 .
$$

Then there exist Yetter-Drinfeld submodules $W_{1} \in \mathcal{Y}_{1}, W_{2} \in \mathcal{Y}_{6}, W_{3} \in \mathcal{Y}_{1}, W_{4} \in \mathcal{Y}_{3}$, $W_{5} \in \mathcal{Y}_{2}$, and $W_{6} \in \mathcal{Y}_{3}$ of $\mathfrak{B}(V \oplus W)$ of degrees $\alpha_{1}, 2 \alpha_{1}+\alpha_{2}, 3 \alpha_{1}+2 \alpha_{2}, 4 \alpha_{1}+3 \alpha_{2}$, $\alpha_{1}+\alpha_{2}$, and $\alpha_{2}$, respectively, such that

$$
\mathfrak{B}(V \oplus W) \simeq \mathfrak{B}\left(W_{6}\right) \otimes \mathfrak{B}\left(W_{5}\right) \otimes \cdots \otimes \mathfrak{B}\left(W_{1}\right)
$$

as $\mathbb{N}_{0}^{2}$-graded vector spaces in $G_{G}^{G} \mathcal{Y D}$. In particular, the Hilbert series of $\mathfrak{B}(V \oplus W)$ is

$$
\mathcal{H}\left(t_{1}, t_{2}\right)=(2)_{t_{2}}(3)_{t_{1} t_{2}}(4)_{t_{1} t_{2}}(6)_{t_{1} t_{2}}(6)_{t_{1}^{2} t_{2}^{2}}(2)_{t_{1}^{4} t_{2}^{3}}(2)_{t_{1}^{3} t_{2}^{2}}^{2}(3)_{t_{1}^{3} t_{2}^{2}}(3)_{t_{1}^{2} t_{2}}^{2}(2)_{t_{1}}^{2}(3)_{t_{1}}
$$

and $\operatorname{dim} \mathfrak{B}(V \oplus W)=2^{10} 3^{7}=2239488$.

Proof. Let $V^{\prime}, W^{\prime}$ be Yetter-Drinfeld modules over $G$. If $\left(V^{\prime}, W^{\prime}\right) \in \wp_{5}$, then $V^{\prime} \in \mathcal{Y}_{1}$ and $W^{\prime} \in \mathcal{Y}_{3}$ by Tables 3 and 4 . Similarly, if $\left(V^{\prime}, W^{\prime}\right) \in \wp_{5}^{\prime}$, then $V^{\prime} \in \mathcal{Y}_{1}$ and $W^{\prime} \in \mathcal{Y}_{6}$, and if $\left(V^{\prime}, W^{\prime}\right) \in \wp_{5}^{\prime \prime}$, then $V^{\prime} \in \mathcal{Y}_{2}$ and $W^{\prime} \in \mathcal{Y}_{3}$.

By assumption, $(V, W) \in \wp_{5}$. Lemmas 7.5 7.10 and Lemma 8.5 imply that the pair $(V, W)$ admits all reflections and that the set of real roots $\Delta^{\mathrm{re}(V, W)}$ is finite. More precisely, $\left|\Delta_{+}^{\mathrm{re}(V, W)}\right|=6$. Hence, by [24, Cor. 6.16], there exist absolutely simple Yetter-Drinfeld submodules $W_{i} \in{ }_{G}^{G} \mathcal{Y} \mathcal{D}$ of $\mathfrak{B}(V \oplus W)$ with $1 \leq i \leq 6$, such that

$$
\mathfrak{B}(V \oplus W) \simeq \mathfrak{B}\left(W_{6}\right) \otimes \mathfrak{B}\left(W_{5}\right) \otimes \cdots \otimes \mathfrak{B}\left(W_{1}\right)
$$

By the same reference, we may also assume that

$$
\operatorname{deg} W_{2 i+1}=\left(s_{1} s_{2}\right)^{i}\left(\alpha_{1}\right) \text { and } \operatorname{deg} W_{2 i+2}=\left(s_{1} s_{2}\right)^{i} s_{1}\left(\alpha_{2}\right)
$$

for all $0 \leq i \leq 2$, and if $1 \leq k \leq 6$ and $\operatorname{deg} W_{k}=s_{i_{1}} \cdots s_{i_{m}}\left(\alpha_{j}\right)$ for some $m \geq$ 0 and $i_{1}, \ldots, i_{m}, j \in\{1,2\}$, then $W_{k}$ is isomorphic in ${ }_{G}^{G} \mathcal{Y} \mathcal{D}$ to the $j$-th entry of $R_{i_{m}} \cdots R_{i_{1}}(V, W)$. Since $\Delta_{+}^{\mathrm{re}(V, W)}$ consists of the roots

$$
\begin{gathered}
s_{1}\left(\alpha_{2}\right)=2 \alpha_{1}+\alpha_{2}, \quad s_{1} s_{2}\left(\alpha_{1}\right)=3 \alpha_{1}+2 \alpha_{2}, \quad s_{1} s_{2} s_{1}\left(\alpha_{2}\right)=4 \alpha_{1}+3 \alpha_{2}, \\
s_{1} s_{2} s_{1} s_{2}\left(\alpha_{1}\right)=\alpha_{1}+\alpha_{2}, \quad s_{1} s_{2} s_{1} s_{2} s_{1}\left(\alpha_{2}\right)=\alpha_{2}, \quad \text { and } \quad \alpha_{1},
\end{gathered}
$$


and since

$$
\begin{gathered}
R_{1}(V, W) \in \wp_{5}^{\prime}, \quad R_{2} R_{1}(V, W) \in \wp_{5}^{\prime}, \quad R_{1} R_{2} R_{1}(V, W) \in \wp_{5}, \\
R_{2} R_{1} R_{2} R_{1}(V, W) \in \wp_{5}^{\prime \prime}, \quad R_{1} R_{2} R_{1} R_{2} R_{1}(V, W) \in \wp_{5}^{\prime \prime},
\end{gathered}
$$

the first paragraph of the proof implies that $W_{1} \in \mathcal{Y}_{1}, W_{2} \in \mathcal{Y}_{6}, W_{3} \in \mathcal{Y}_{1}, W_{4} \in \mathcal{Y}_{3}$, $W_{5} \in \mathcal{Y}_{2}, W_{6} \in \mathcal{Y}_{3}$. Finally, a direct calculation using Table 4 yields the Hilbert series of $\mathfrak{B}(V \oplus W)$.

With similar proofs, or by applying the reflections $R_{1}$ and $R_{2}$ to pairs in $\wp_{5}$, one obtains also the following two theorems on Nichols algebras of $V \oplus W$, where $(V, W) \in \wp_{5}^{\prime}$ or $(V, W) \in \wp_{5}^{\prime \prime}$.

Theorem 8.7. Let $V, W \in G_{G}^{G} \mathcal{Y D}$ such that $V \simeq M(g, \rho)$, where $\rho$ is a character of $G^{g}$, and $W \simeq M(\epsilon z, \sigma)$, where $\sigma$ is a character of $G^{\epsilon}$. Assume that char $\mathbb{K}=2$ and $(V, W) \in \wp_{5}^{\prime}$, that is,

$$
\rho(g)=1, \quad \sigma(z)=\sigma(\epsilon), \quad(3)_{\sigma(\epsilon)}=0, \quad \rho\left(z^{2}\right) \sigma\left(\epsilon g^{2}\right)=1 .
$$

Then there exist Yetter-Drinfeld submodules $W_{1} \in \mathcal{Y}_{1}, W_{2} \in \mathcal{Y}_{3}, W_{3} \in \mathcal{Y}_{2}, W_{4} \in \mathcal{Y}_{3}$, $W_{5} \in \mathcal{Y}_{1}$, and $W_{6} \in \mathcal{Y}_{6}$ of $\mathfrak{B}(V \oplus W)$ of degrees $\alpha_{1}, 2 \alpha_{1}+\alpha_{2}, \alpha_{1}+\alpha_{2}, 2 \alpha_{1}+3 \alpha_{2}$, $\alpha_{1}+2 \alpha_{2}$, and $\alpha_{2}$, respectively, such that

$$
\mathfrak{B}(V \oplus W) \simeq \mathfrak{B}\left(W_{6}\right) \otimes \mathfrak{B}\left(W_{5}\right) \otimes \cdots \otimes \mathfrak{B}\left(W_{1}\right)
$$

as $\mathbb{N}_{0}^{2}$-graded vector spaces in ${ }_{G}^{G} \mathcal{Y} \mathcal{D}$. In particular, the Hilbert series of $\mathfrak{B}(V \oplus W)$ is

$$
\mathcal{H}\left(t_{1}, t_{2}\right)=(3)_{t_{2}}^{2}(2)_{t_{1} t_{2}^{2}}^{2}(3)_{t_{1} t_{2}^{2}}(2)_{t_{1}^{2} t_{2}^{3}}(3)_{t_{1} t_{2}}(4)_{t_{1} t_{2}}(6)_{t_{1} t_{2}}(6)_{t_{1}^{2} t_{2}^{2}}(2)_{t_{1}^{2} t_{2}}(2)_{t_{1}}^{2}(3)_{t_{1}}
$$

and $\operatorname{dim} \mathfrak{B}(V \oplus W)=2^{10} 3^{7}=2239488$.

Theorem 8.8. Let $V, W \in{ }_{G}^{G} \mathcal{Y D}$ such that $V \simeq M(g, \rho)$, where $\rho$ is a character of $G^{g}$, and $W \simeq M(z, \sigma)$, where $\sigma$ is a character of $G$. Assume that char $\mathbb{K}=2$ and $(V, W) \in \wp_{5}^{\prime \prime}$, that is,

$$
(3)_{\rho(g)}=0, \quad \sigma(z)=1, \quad \rho(g z) \sigma(g)=1 .
$$

Then there exist Yetter-Drinfeld submodules $W_{1} \in \mathcal{Y}_{2}, W_{2} \in \mathcal{Y}_{3}, W_{3} \in \mathcal{Y}_{1}, W_{4} \in \mathcal{Y}_{6}$, $W_{5} \in \mathcal{Y}_{1}$, and $W_{6} \in \mathcal{Y}_{3}$ of $\mathfrak{B}(V \oplus W)$ of degrees $\alpha_{1}, 4 \alpha_{1}+\alpha_{2}, 3 \alpha_{1}+\alpha_{2}, 2 \alpha_{1}+\alpha_{2}$, $\alpha_{1}+\alpha_{2}$, and $\alpha_{2}$, respectively, such that

$$
\mathfrak{B}(V \oplus W) \simeq \mathfrak{B}\left(W_{6}\right) \otimes \mathfrak{B}\left(W_{5}\right) \otimes \cdots \otimes \mathfrak{B}\left(W_{1}\right)
$$

as $\mathbb{N}_{0}^{2}$-graded vector spaces in $G_{G}^{G} \mathcal{Y} \mathcal{D}$. In particular, the Hilbert series of $\mathfrak{B}(V \oplus W)$ is

$$
\mathcal{H}\left(t_{1}, t_{2}\right)=(2)_{t_{2}}(2)_{t_{1} t_{2}}^{2}(3)_{t_{1} t_{2}}(3)_{t_{1}^{2} t_{2}}^{2}(2)_{t_{1}^{3} t_{2}}^{2}(3)_{t_{1}^{3} t_{2}}(2)_{t_{1}^{4} t_{2}}(3)_{t_{1}}(4)_{t_{1}}(6)_{t_{1}}(6)_{t_{1}^{2}}
$$

and $\operatorname{dim} \mathfrak{B}(V \oplus W)=2^{10} 3^{7}=2239488$. 


\section{Proof of Theorem 2.1}

Here we prove the main result of the paper, which is Theorem 2.1.

First we prove $(1) \Rightarrow(2)$. Since $\operatorname{dim} \mathfrak{B}(V \oplus W)<\infty$, the pair $(V, W)$ admits all reflections by [5, Cor. 3.18] and the Weyl groupoid is finite by [5, Prop. 3.23].

Now we prove simultaneously $(2) \Rightarrow(3)$ and $(3) \Rightarrow(1)$. By [25, Thm. 7.3], the quandle $\operatorname{supp}(V \oplus W)$ is isomorphic to

$$
Z_{2}^{2,2}, Z_{3}^{3,1}, Z_{3}^{3,2}, Z_{4}^{4,2} \text { or } Z_{T}^{4,1}
$$

and $G$ is a non-abelian epimorphic image of its enveloping group:

\begin{tabular}{c|ccccc} 
Quandle & $Z_{T}^{4,1}$ & $Z_{2}^{2,2}$ & $Z_{3}^{3,1}$ & $Z_{3}^{3,2}$ & $Z_{4}^{4,2}$ \\
\hline Enveloping group & $T$ & $\Gamma_{2}$ & $\Gamma_{3}$ & $\Gamma_{3}$ & $\Gamma_{4}$
\end{tabular}

We consider each case separately. Suppose first that $G$ is an epimorphic image of $\Gamma_{2}$ and $\operatorname{supp}(V \oplus W) \simeq Z_{2}^{2,2}$. Hence, by [22, $\left.\S 4\right]$, we may assume that $V \simeq M(g, \rho)$ and $W \simeq M(h, \sigma)$. Now the claim follows from [22, Thm. 4.9].

Now assume that $G$ is a non-abelian epimorphic image of $\Gamma_{3}$. We first prove that (2) implies (3). By [26, Prop. 4.3], there is a pair $\left(V^{\prime}, W^{\prime}\right)$ of absolutely simple Yetter-Drinfeld modules over $G$, which represents an object of the Weyl groupoid of $(V, W)$, such that the Cartan matrix of $\left(V^{\prime}, W^{\prime}\right)$ is of finite type. The list of examples in Subsection 1.4 contains precisely the pairs in the classes $\wp_{i}$ for $1 \leq i \leq 5$ and $\wp_{5}^{\prime}, \wp_{5}^{\prime \prime}$. Hence this list is stable under reflections by Lemmas 7.3, 17.4, and 7.5 7.10, Thus we may assume that the Cartan matrix of $(V, W)$ is of finite type. Now (3) follows from Proposition 7.2 .

The implication $(3) \Rightarrow(1)$ follows from Theorems [8.1, 8.2, 8.3, 8.4, 8.6, 8.7, and 8.8 .

Assume now that $G$ is a non-abelian epimorphic image of $\Gamma_{4}$, but not of $\Gamma_{2}$, and that $\operatorname{supp}(V \oplus W) \simeq Z_{4}^{4,2}$. Without loss of generality we may assume that $|\operatorname{supp} V|=2$ and $|\operatorname{supp} W|=4$. Let $h \in \operatorname{supp} V, g \in \operatorname{supp} W$, and $\epsilon=h g h^{-1} g^{-1}$. Then $g^{G}=\left\{g, \epsilon g, \epsilon^{2} g, \epsilon^{3} g\right\}$ and $h^{G}=\left\{h, \epsilon^{-1} h\right\}$. Since $\operatorname{supp}(V \oplus W)$ generates $G$, we conclude that $G$ is generated by $g$ and $h$, and $V \simeq M(h, \rho)$ and $W \simeq M(g, \sigma)$ for some character $\rho$ of $G^{h}$ and some character $\sigma$ of $G^{g}$. Then the implications $(2) \Rightarrow(3)$ and $(3) \Rightarrow(1)$ follow from [25, Thm. 5.4].

Finally, suppose that $G$ is an epimorphic image of $T$ and $\operatorname{supp}(V \oplus W) \simeq Z_{T}^{4,1}$. We may assume that $|\operatorname{supp} V|=1$ and $|\operatorname{supp} W|=4$. Let $z \in \operatorname{supp} V$ and $x_{1} \in \operatorname{supp} W$. Then $z$ and $x_{1}^{G}$ generate $G$. Choose $x_{2} \in x_{1}^{G} \backslash\left\{x_{1}\right\}$. Then $T \rightarrow G, \chi_{1} \mapsto x_{1}, \chi_{2} \mapsto x_{2}$, $\zeta \mapsto z$ is an epimorphism of groups, and $V \simeq M(z, \rho)$ and $W \simeq M\left(x_{1}, \sigma\right)$ for some absolutely irreducible representations $\rho$ of $G$ and $\sigma$ of $G^{x_{1}}$. Then the implications $(2) \Rightarrow(3)$ and $(3) \Rightarrow(1)$ follow from [25, Thm. 2.8].

Acknowledgments. Leandro Vendramin was supported by Conicet, UBACyT 20020110300037 and the Alexander von Humboldt Foundation. 


\section{REFERENCES}

[1] N. Andruskiewitsch.: About finite dimensional Hopf algebras. In Quantum symmetries in theoretical physics and mathematics (Bariloche, 2000), volume 294 of Contemp. Math., pages 1-57. Amer. Math. Soc., Providence, RI, 2002.

[2] Andruskiewitsch, N., Fantino, F., García, G. A., Vendramin, L.: On Nichols algebras associated to simple racks. In Groups, algebras and applications, volume 537 of Contemp. Math., pages 31-56. Amer. Math. Soc., Providence, RI, 2011.

[3] Andruskiewitsch, N., Fantino, F., Graña, M., Vendramin, L.: Finite-dimensional pointed Hopf algebras with alternating groups are trivial. Ann. Mat. Pura Appl. 190(2), 225-245 (2011)

[4] Andruskiewitsch, N., Fantino, F., Graña, M., Vendramin, L.: Pointed Hopf algebras over the sporadic simple groups. J. Algebra 325, 305-320 (2011)

[5] Andruskiewitsch, N., Heckenberger, I., Schneider, H.-J.: The Nichols algebra of a semisimple Yetter-Drinfeld module. Amer. J. Math. 132(6), 1493-1547 (2010)

[6] Andruskiewitsch, N., Schneider, H.-J.: Lifting of quantum linear spaces and pointed Hopf algebras of order $p^{3}$. J. Algebra 209(2), 658-691 (1998)

[7] Andruskiewitsch, N., Schneider, H.-J.: Pointed Hopf algebras. In New directions in Hopf algebras, volume 43 of Math. Sci. Res. Inst. Publ., pages 1-68. Cambridge Univ. Press, Cambridge, 2002.

[8] Andruskiewitsch, N., Schneider, H.-J.: On the classification of finite-dimensional pointed Hopf algebras. Ann. of Math. 171(1), 375-417 (2010)

[9] Angiono, I.: A presentation by generators and relations of Nichols algebras of diagonal type and convex orders on root systems. Accepted for publication in J. Europ. Math. Soc., arXiv:1008.4144.

[10] Angiono, I.: On Nichols algebras of diagonal type. J. Reine Angew. Math. 683, 189-251 (2013)

[11] Bazlov, Y.: Nichols-Woronowicz algebra model for Schubert calculus on Coxeter groups. J. Algebra 297(2), 372-399 (2006)

[12] Cuntz, M., Heckenberger, I.: Weyl groupoids of rank two and continued fractions. Algebra Number Theory 3(3), 317-340 (2009)

[13] Cuntz, M., Heckenberger, I.: Weyl groupoids with at most three objects. J. Pure Appl. Algebra 213(6), 1112-1128 (2009)

[14] Fomin, S., Kirillov, A. N.: Quadratic algebras, Dunkl elements, and Schubert calculus. In Advances in geometry, volume 172 of Progr. Math., pages 147-182. Birkhäuser Boston, Boston, MA, 1999.

[15] Gaberdiel, M. R.: An algebraic approach to logarithmic conformal field theory. In Proceedings of the School and Workshop on Logarithmic Conformal Field Theory and its Applications (Tehran, 2001), volume 18, pages 4593-4638, 2003.

[16] Graña, M.: A freeness theorem for Nichols algebras. J. Algebra 231(1), 235-257 (2000)

[17] Graña, M.: On Nichols algebras of low dimension. In New trends in Hopf algebra theory (La Falda, 1999), volume 267 of Contemp. Math., pages 111-134. Amer. Math. Soc., Providence, RI, 2000.

[18] Graña, M., Heckenberger, I., Vendramin, L.: Nichols algebras of group type with many quadratic relations. Adv. Math. 227(5), 1956-1989 (2011)

[19] Heckenberger, I.: The Weyl groupoid of a Nichols algebra of diagonal type. Invent. Math. 164(1), 175-188 (2006)

[20] Heckenberger, I.: Classification of arithmetic root systems. Adv. Math. 220(1), 59-124 (2009)

[21] Heckenberger, I., Lochmann, A., Vendramin, L.: Braided racks, Hurwitz actions and Nichols algebras with many cubic relations. Transform. Groups 17(1), 157-194 (2012)

[22] Heckenberger, I., Schneider, H.-J.: Nichols algebras over groups with finite root system of rank two I. J. Algebra 324(11), 3090-3114 (2010)

[23] Heckenberger, I., Schneider, H.-J.: Root systems and Weyl groupoids for Nichols algebras. Proc. Lond. Math. Soc. 101(3), 623-654 (2010)

[24] Heckenberger, I., Schneider, H.-J.: Right coideal subalgebras of Nichols algebras and the Duflo order on the Weyl groupoid. Israel J. Math. 197(1), 139-187 (2013) 
[25] Heckenberger, I., Vendramin, L.: Nichols algebras over groups with finite root system of rank two III. arXiv:1309.4634, 2013.

[26] Heckenberger, I., Vendramin, L.: Nichols algebras over groups with finite root system of rank two II. Accepted for publication in J. Group Theory, DOI:10.1515/jgth-2014-0024.

[27] Lusztig, G.: Introduction to Quantum Groups. Modern Birkhäuser Classics. Birkhäuser/Springer, New York, 2010. Reprint of the 1994 edition.

[28] Majid, S.: Noncommutative differentials and Yang-Mills on permutation groups $S_{n}$. In Hopf algebras in noncommutative geometry and physics, volume 239 of Lecture Notes in Pure and Appl. Math., pages 189-213. Dekker, New York, 2005.

[29] Milinski, A., Schneider, H.-J.: Pointed indecomposable Hopf algebras over Coxeter groups. In New trends in Hopf algebra theory (La Falda, 1999), volume 267 of Contemp. Math., pages 215-236. Amer. Math. Soc., Providence, RI, 2000.

[30] Müller, E.: Some topics on Frobenius-Lusztig kernels. I, II. J. Algebra 206(2), 624-658, 659$681(1998)$

[31] Nichols, W. D.: Bialgebras of type one. Comm. Algebra 6(15), 1521-1552 (1978)

[32] Rosso, M.: Quantum groups and quantum shuffles. Invent. Math. 133(2), 399-416 (1998)

[33] Semikhatov, A.M., Tipunin, I. Y.: The Nichols algebra of screenings. Commun. Contemp. Math. 14(4), 1250029, 66 (2012)

[34] Woronowicz, S.L.: Compact matrix pseudogroups. Comm. Math. Phys. 111(4), 613-665 (1987)

[35] Woronowicz, S. L.: Differential calculus on compact matrix pseudogroups (quantum groups). Comm. Math. Phys. 122(1), 125-170 (1989)

I. Heckenberger: Philipps-Universität Marburg, FB Mathematik und Informatik, Hans-Meerwein-Strasse, 35032 Marburg, Germany.

E-mail address: heckenberger@mathematik. uni-marburg.de

L. Vendramin: Departamento de Matemática, FCen, Universidad de Buenos Aires, Pabellón 1, Ciudad Universitaria (1428), Buenos Aires, Argentina.

E-mail address: lvendramin@dm.uba.ar 